THE TAOS PROJECT: UPPER BOUNDS ON THE POPULATION OF SMALL KUIPER BELT OBJECTS AND TESTS OF MODELS OF FORMATION AND EVOLUTION OF THE OUTER SOLAR SYSTEM

This article has been downloaded from IOPscience. Please scroll down to see the full text article.

2010 The Astronomical Journal 1391499

(http://iopscience.iop.org/1538-3881/139/4/1499)

The Table of Contents and more related content is available

Download details:

IP Address: 131.215.193.213

The article was downloaded on 26/03/2010 at 21:30

Please note that terms and conditions apply. 


\title{
THE TAOS PROJECT: UPPER BOUNDS ON THE POPULATION OF SMALL KUIPER BELT OBJECTS AND TESTS OF MODELS OF FORMATION AND EVOLUTION OF THE OUTER SOLAR SYSTEM
}

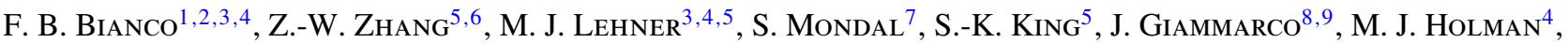 \\ N. K. Coehlo ${ }^{10}$, J.-H. Wang ${ }^{5,6}$, C. AlCoCK ${ }^{4}$, T. Axelrod ${ }^{11}$, Y.-I. Byun ${ }^{12}$, W. P. Chen ${ }^{6}$, K. H. CoOK ${ }^{13}$, R. Dave ${ }^{14}$, \\ I. De Pater ${ }^{15}$, D.-W. Kim ${ }^{12,14}$, T. LeE $^{5}$, H.-C. Lin $^{5}$, J. J. Lissauer ${ }^{16}$, S. L. Marshall ${ }^{17}$, P. Protopapas ${ }^{4,14}$, J. A. Rice ${ }^{10}$, \\ M. E. SCHWAMB ${ }^{18}$, S.-Y. WANG ${ }^{5}$, AND C.-Y. WEN ${ }^{5}$ \\ ${ }^{1}$ Department of Physics, University of California Santa Barbara, Mail Code 9530, Santa Barbara, CA 93106-9530, USA; fbianco@1cogt.net \\ ${ }^{2}$ Las Cumbres Observatory Global Telescope Network, Inc. 6740 Cortona Drive, Suite 102, Santa Barbara, CA 93117, USA \\ ${ }^{3}$ Department of Physics and Astronomy, University of Pennsylvania, 209 South 33rd Street, Philadelphia, PA 19104, USA \\ ${ }^{4}$ Harvard-Smithsonian Center for Astrophysics, 60 Garden Street, Cambridge, MA 02138, USA \\ ${ }^{5}$ Institute of Astronomy and Astrophysics, Academia Sinica, P.O. Box 23-141, Taipei 10617, Taiwan \\ ${ }^{6}$ Institute of Astronomy, National Central University, 300 Jhongda Road, Jhongli 32054, Taiwan \\ ${ }^{7}$ Aryabhatta Research Institute of Observational Sciences (ARIES), Manora Peak, Nainital 263 129, India \\ ${ }^{8}$ Department of Astronomy and Physics, Eastern University, 1300 Eagle Road, Saint Davids, PA 19087, USA \\ ${ }^{9}$ Department of Physics, Villanova University, 800 Lancaster Avenue, Villanova, PA 19085, USA \\ ${ }^{10}$ Department of Statistics, University of California-Berkeley, 367 Evans Hall, Berkeley, CA 94720, USA \\ ${ }^{11}$ Steward Observatory, 933 North Cherry Avenue, Room N204, Tucson, AZ 85721, USA \\ 12 Department of Astronomy, Yonsei University, 134 Shinchon, Seoul 120-749, Republic of Korea \\ ${ }^{13}$ Institute for Geophysics and Planetary Physics, Lawrence Livermore National Laboratory, Livermore, CA 94550, USA \\ ${ }^{14}$ Initiative in Innovative Computing at Harvard, 60 Oxford Street, Cambridge, MA 02138, USA \\ ${ }^{15}$ Department of Astronomy, University of California-Berkeley, 601 Campbell Hall, Berkeley, CA 94720, USA \\ ${ }^{16}$ Space Science and Astrobiology Division 245-3, NASA Ames Research Center, Moffett Field, CA 94035, USA \\ ${ }^{17}$ Kavli Institute for Particle Astrophysics and Cosmology, 2575 Sand Hill Road, MS 29, Menlo Park, CA 94025, USA \\ ${ }^{18}$ Division of Geological and Planetary Sciences, California Institute of Technology, 1201 East California Boulevard, Pasadena, CA 91125, USA \\ Received 2009 July 7; accepted 2010 February 4; published 2010 March 9
}

\begin{abstract}
We have analyzed the first 3.75 years of data from the Taiwanese American Occultation Survey (TAOS). TAOS monitors bright stars to search for occultations by Kuiper Belt objects (KBOs). This data set comprises $5 \times 10^{5}$ star hours of multi-telescope photometric data taken at 4 or $5 \mathrm{~Hz}$. No events consistent with KBO occultations were found in this data set. We compute the number of events expected for the Kuiper Belt formation and evolution models of Pan \& Sari, Kenyon \& Bromley, Benavidez \& Campo Bagatin, and Fraser. A comparison with the upper limits we derive from our data constrains the parameter space of these models. This is the first detailed comparison of models of the KBO size distribution with data from an occultation survey. Our results suggest that the KBO population is composed of objects with low internal strength and that planetary migration played a role in the shaping of the size distribution.
\end{abstract}

Key words: Kuiper belt: general - occultations

\section{INTRODUCTION}

The Kuiper Belt has been shaped by accretion and disruption processes throughout the history of the solar system. With small orbital eccentricities, the relative velocities of the objects in the early Kuiper Belt were sufficiently low to allow accretion processes to form kilometer and much larger objects. Later, the velocity dispersion increased, possibly as the KBO population was stirred up by the gravitational effects of the larger planets and planetoids. Only large objects were then able to continue growing through impacts, whereas collisions among smaller bodies resulted in disruption. The details of these processes depend on the internal strength of the KBOs and on the orbital and dynamical evolution of the gas giant planets. The size distribution of KBOs, therefore, contains information on the internal structure and composition of the KBOs-and hence information on the location and epoch in which they formedand on planetary migration (Kenyon et al. 2008, and references therein). Direct observations have detected KBOs as faint as magnitude $R \sim 28.2$ (Bernstein et al. 2004), which corresponds to a diameter of about $27 \mathrm{~km}$ assuming a $4 \%$ albedo. The large end side of the KBO size distribution can therefore be characterized through its brightness distribution. The latter is well described by a power law $\Sigma(<R)=10^{\alpha\left(R-R_{0}\right)}$, with an index $\alpha=0.6$ and $R_{0}=23$ (Fraser \& Kavelaars 2009; Fuentes $\&$ Holman 2008) for objects brighter than about $R=25$, or $D \sim 100 \mathrm{~km}$. This is the region of the size spectrum that reflects the early history of agglomeration. Kenyon \& Windhorst (2001) pointed out that the intensity of the infrared Zodiacal Background sets limits on the extrapolation of a straight power law to smaller sizes. The relatively shallow size distribution of Jupiter Family Comets (JFCs; Tancredi et al. 2006), which are believed to originate in the Kuiper Belt, and the cratering of Triton observed by Voyager 2 (Stern 1996), all point to a flatter distribution for small KBOs. ${ }^{19}$ In 2004 evidence surfaced that a break in the power law occurs at a diameter larger than $10 \mathrm{~km}$ : Bernstein et al. (2004) conducted deep Hubble Space Telescope (HST) observations with the Advanced Camera for Surveys which led to the discovery of only three new objects fainter than $R=26$, about $4 \%$ of the number expected from a single power-law distribution extrapolated to $10 \mathrm{~km}$. While this work remains the state of the art for deep direct surveys of the outer solar system, recent campaigns have observed many more faint objects down to magnitude $R=27$, which with the assumption of a $4 \%$ albedo corresponds to about $40 \mathrm{~km}$ in

\footnotetext{
19 The relationship between the cratering of Triton and the Kuiper Belt size
} distribution is questioned by Schenk \& Zahnle (2007). 
diameter ${ }^{20}$ (Fraser \& Kavelaars 2008, 2009; Fuentes \& Holman 2008; Fuentes et al. 2009). These recent data allowed them to locate a break in the power-law size distribution at diameters $30 \lesssim D \lesssim 120 \mathrm{~km}$.

The region of the size spectrum between tens of kilometers and meters in diameter is particularly interesting as models predict here the occurrence of transitions between different regimes where the binding energy of KBOs is dominated either by gravity or internal strength. These transitions would leave a signature in the size distribution (Pan \& Sari 2005, Kenyon \& Bromley 2004, Benavidez \& Campo Bagatin 2009, and references therein). Occultation surveys allow us to reach farther than the current limits of direct observations, and into this region of interest. These surveys monitor background stars in order to detect the chance alignment of a KBO with a target star, which would generate a variation in the observed flux of the star. At distances in the outer solar system (tens to thousands of $\mathrm{AU}$ ) the signature left in a light curve by the transits of $D \sim 1 \mathrm{~km}$ objects is dominated by diffraction. This technique requires high frequency photometric time series as the timescale for an occultation by an outer solar system object is a fraction of a second (Roques \& Moncuquet 2000; Nihei et al. 2007; Bickerton et al. 2009). A few such surveys have been attempted in the past several years and have recently started reporting results: e.g., Roques et al. (2006), Chang et al. (2007), Bickerton et al. (2008), Liu et al. (2008), Zhang et al. (2008, hereinafter Z08), Bianco et al. (2009), and Wang et al. (2009). None of these surveys have claimed detections in the Kuiper Belt; upper limits have thus been placed on the number density of KBOs in the sky.

Bickerton et al. (2008) set an upper limit to the sky density of KBOs of $\Sigma_{N}(D \geqslant 1 \mathrm{~km}) \leqslant 2.8 \times 10^{9} \mathrm{deg}^{-2}$ using the $40 \mathrm{~Hz}$ data from their own survey as well as the $45 \mathrm{~Hz}$ data from Roques et al. (2006) and the X-ray data from Chang et al. (2007). Bianco et al. (2009) carried out a $30 \mathrm{~Hz}$ survey with Megacam at the MMT setting a more stringent limit of $\Sigma_{N}(D \geqslant 1 \mathrm{~km}) \leqslant 2.0 \times$ $10^{8} \mathrm{deg}^{-2}$ and a limit of $\Sigma_{N}(D \geqslant 0.7 \mathrm{~km}) \leqslant 4.8 \times 10^{8} \mathrm{deg}^{-2}$. Wang et al. (2009) reported preliminary analysis of videomode engineering data taken with the Pan-STARRS system.

Recently Schlichting et al. (2009) reported the detection of a candidate occultation event consistent with a $D \sim 1 \mathrm{~km}$ $\mathrm{KBO}$ in the analysis of archival guiding data from $H S T$, and an estimate of the sky density of KBOs of $\Sigma_{N}(D \geqslant 0.5 \mathrm{~km})=$ $2.1_{-1.2}^{+4.8} \times 10^{7} \mathrm{deg}^{-2}$.

The Taiwanese American Occultation Survey (TAOS) has been operating since 2005 with two, three, and now four telescopes simultaneously taking stellar photometry at $5 \mathrm{~Hz}^{21}$ The analysis of the first two years of TAOS reported no detections (Z08) and an upper limit was derived to the slope of the small size end of the size spectrum. The TAOS system is described in detail in Lehner et al. (2009). Using $50 \mathrm{~cm}$ aperture robotic telescopes in simultaneous observations and observing with the relatively low cadence compared to the aforementioned occultation surveys, TAOS was designed to address the $\mathrm{km}$ size region of the KBO size spectrum. We will show here that the marginal sensitivity to subkilometer objects is more than compensated by the very large exposure of our star targets. Here we consider the first 3.75 years of TAOS data, a significantly larger data set than the one explored in Z08. With these data

\footnotetext{
20 The magnitude of KBOs is converted into diameter by assuming a nominal $4 \%$ albedo throughout the paper, note however that Fraser \& Kavelaars (2008, 2009 ) assumed an albedo of $6 \%$ in their work.

21 A small subset of early data was collected at $4 \mathrm{~Hz}$ cadence, comprising about $5 \%$ of the data analyzed in this work.
}

we are able to constrain Kuiper Belt formation and evolution models.

In Section 2, we describe the new data set. In Section 3, we briefly describe our detection algorithms, as well as our efficiency analysis. We also discuss our recovery efficiency and discuss the most productive strategies for TAOS and the other occultation surveys, and, in Section 3.4, we derive the effective coverage of our survey. In Section 4, we derive modelindependent limits to the number of objects in the Kuiper Belt, and we compare our results with those of similar surveys. In Section 5, we briefly describe models for the formation and evolution of the Kuiper Belts and we then derive and discuss constraints to these models. In Section 6, we compare our upper limits to the estimates on the number of KBOs set by dynamical simulations for JFC progenitor populations. Finally, we summarize and discuss our findings in Section 7.

\section{3.75 YEARS OF TAOS DATA}

TAOS is a dedicated survey that observes at a cadence of $5 \mathrm{~Hz}$. The primary scientific goal of the survey is to estimate or set constraints on the number of KBOs in the region of the size spectrum that is currently too small to be observed directly: $D \lesssim 10 \mathrm{~km}$.

Here, we present an expanded analysis of three-telescope TAOS data. ${ }^{22}$ These data consist of photometric measurements of target star fields collected synchronously with all three telescopes. The data set analyzed here was collected between 2005 January and 2008 August. In a previous analysis of a subset of these data, $\mathrm{Z} 08$ reported an upper limit to the size distribution of KBOs under the assumption of a single power law for small KBOs. If one models the size distribution for objects smaller than $D=28 \mathrm{~km}$, the smallest direct observation (Bernstein et al. 2004), as a single power law $d N / d D \propto D^{-q}$, where $N$ is the surface density of objects, the slope of the distribution is limited to $q \leqslant 4.6$.

Throughout the remainder of this paper, a "data run" refers to a set of data collected in an uninterrupted observation of any field. For a single star in the field a set of three light curves belonging to one data run will be referred to as a "light curve set," and each three-telescope measurement, at a single time point, will be referred to as a "triplet." A "star hour" refers to an hour of high-cadence, multi-telescope observations on a single target star.

The data set described in this paper amounts to $5.0 \times 10^{5} \mathrm{star}$ hours, while the data set used in Z08 comprises $1.5 \times 10^{5}$ star hours. The details of this data set, and of the data set published in Z08, are summarized in Table 1 . Over $90 \%$ of our data are collected within $5^{\circ}$ of the ecliptic plane in order to maximize the rate of occultations.

TAOS uses the zipper-mode technique to read out the CCD cameras at high frequency. This method, described in Lehner et al. (2009), enables high-speed observations across the $3 \square^{\circ}$ field of view of the TAOS telescopes, but it artificially increases the crowding of the field and the background. In zipper-mode readout each star in the field is represented in a subsection of the output image — which we call rowblock and which comprises 76 rows for our $5 \mathrm{~Hz}$ data-so that the field of view is entirely imaged in each rowblock. Note that the images of different stars in a rowblock, however, do not necessarily belong to the same

\footnotetext{
22 The fourth telescope, TAOS C, became operational in 2008 August. The results presented in this paper are based on analysis of all of the three-telescope data collected to this point.
} 


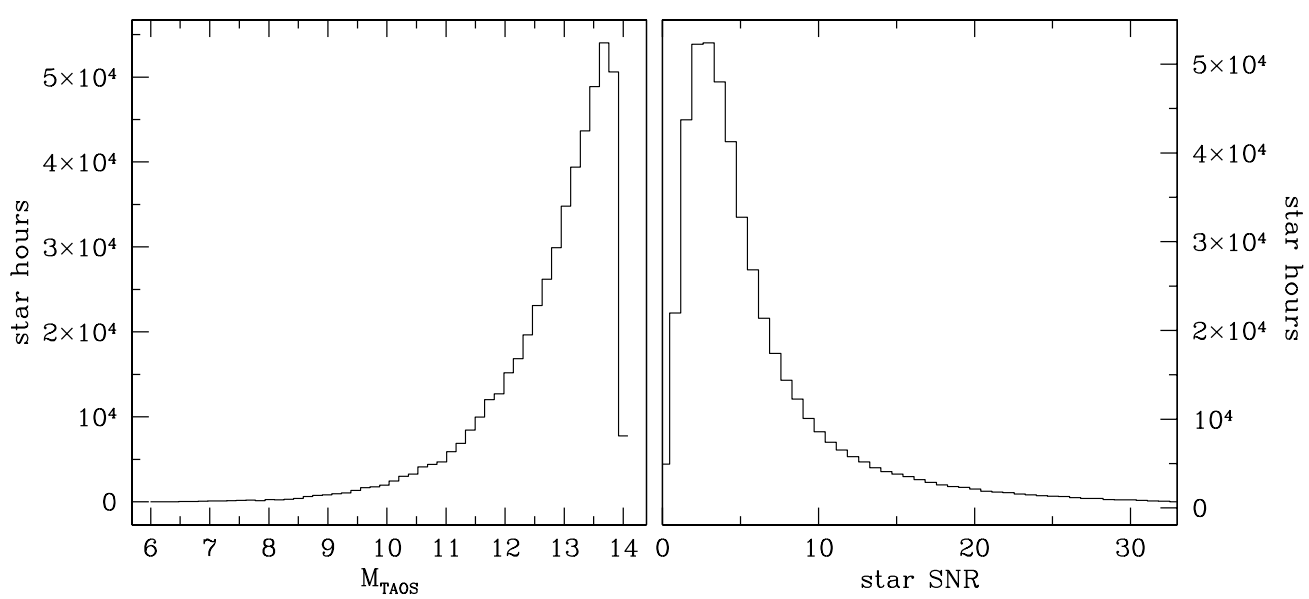

Figure 1. Distribution of magnitudes for the TAOS target stars (bin size $0.16 \mathrm{mag}$, left). $\mathrm{S} / \mathrm{N}$ for the TAOS target stars, averaged over the duration of a run and over the three telescopes (bin size 0.73 , right). A few targets at greater $\mathrm{S} / \mathrm{N}$ and brighter magnitude, amounting to $<5 \%$ of the data, are not shown.

Table 1

Data Set Parameters (Three-telescope Data)

\begin{tabular}{lcc}
\hline \hline \multicolumn{1}{c}{ Parameter } & Z08 & This Work \\
\hline Start date & $2005 \mathrm{Feb}$ & 2005 Feb 7 \\
End date & $2006 \mathrm{Dec} \mathrm{31}$ & 2008 Aug 2 \\
Light-curve sets & 110,554 & 366,083 \\
Exposure (star hours) $_{\text {Triplets }^{\mathrm{a}}}$ & 152,787 & 500,339 \\
& $2.6 \times 10^{9}$ & $9.0 \times 10^{9}$
\end{tabular}

Note. ${ }^{\text {a }}$ Multi-telescope measurements.

epoch. The zipper-mode readout boosts the sky background by a factor of 27 at a $5 \mathrm{~Hz}$ readout rate. This limits the sensitivity of TAOS to stars as faint as $M_{\mathrm{TAOS}}=13.5$, for which a signalto-noise ratio $(\mathrm{S} / \mathrm{N})$ of $\sim 7$ can be achieved in a dark night. The magnitude and $\mathrm{S} / \mathrm{N}$ distributions for the target stars in our survey are shown in Figure 1. On the left panel, the $x$-axis is the TAOS instrumental magnitude $M_{\text {TAOS }}$, which is defined by a regression on the USNO-B magnitudes to be similar to $R_{\mathrm{USNO}}$. The correlation between instrumental magnitude and $\mathrm{S} / \mathrm{N}$ is shown in Figure 2. The scatter in the relationship between $\mathrm{S} / \mathrm{N}$ and $M_{\mathrm{TAOS}}$ is due to both changes in the sky background and in the weather conditions, and to different degrees of crowding in the fields. In Figure 3, left, we show the number of star hours at different angles from opposition. The top scale indicates the velocity of a KBO at the center of a field at this elongation. We cover a large range of opposition angles; our field selection algorithm favors ecliptic fields near zenith. Most angles are positive because the weather at the site tends to improve after midnight. The right panel of Figure 3 shows the distribution of ecliptic latitude of our data. The effects of the angle from opposition on our efficiency and event rate, as well as the efficiency as a function of magnitude and crowding are discussed further in Section 3.3.

\section{ANALYSIS}

The first step in the analysis is the photometric reduction of the zipper mode images in the data set. A custom aperture photometry package (Zhang et al. 2009) is used to measure the brightness of each star at each epoch, and the resulting series of flux measurements is then assembled into light curves for subsequent analysis, which is described in the following subsections. In Section 3.1, we describe our detection algorithm

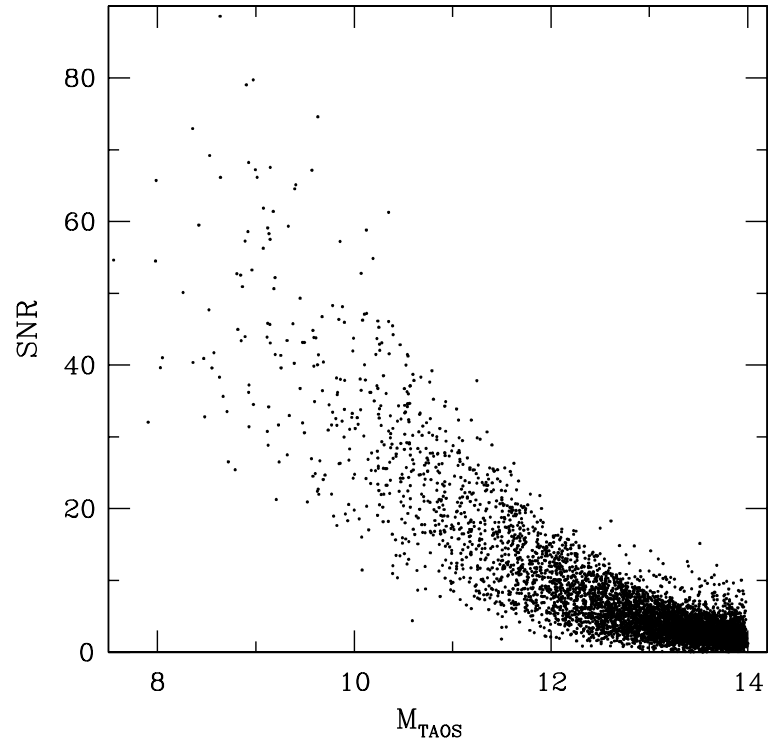

Figure 2. $\mathrm{S} / \mathrm{N}$ vs. TAOS instrumental magnitude $M_{\mathrm{TAOS}}$; only a random sample of $1 \%$ of all stars is shown for clarity.

and the rejection of false positives. We then describe the efficiency tests. In Section 3.2, we describe how we identify the angular size of our target stars. In Section 3.3, we show how we simulate and implant occultation events in our light curves, and test the behavior of our efficiency as a function of various parameters relative to the occultations and to the observing strategy. Finally, we can derive the effective coverage of our survey (Section 3.4).

\subsection{Event Detection and False Positive Rejection}

The Fresnel scale is defined as $F=(\lambda \Delta / 2)^{\frac{1}{2}}$ where $\lambda$ is the wavelength of observation and $\Delta$ the distance to the occulter (Roques et al. 1987; Born \& Wolf 1980). For optical observations at the distance of the Kuiper Belt (about $43 \mathrm{AU}$ ) the Fresnel scale is $F \approx 1.4 \mathrm{~km}$. Occultation events will therefore exhibit significant diffraction effects. Occultations are manifested in the light curve of an observed star as an alternation of bright and dark features, typically with an overall suppression of the flux. Theoretical occultation light curves are shown in Figure 4. The signature of an occultation by a KBO of subkilometer size has a duration of about 

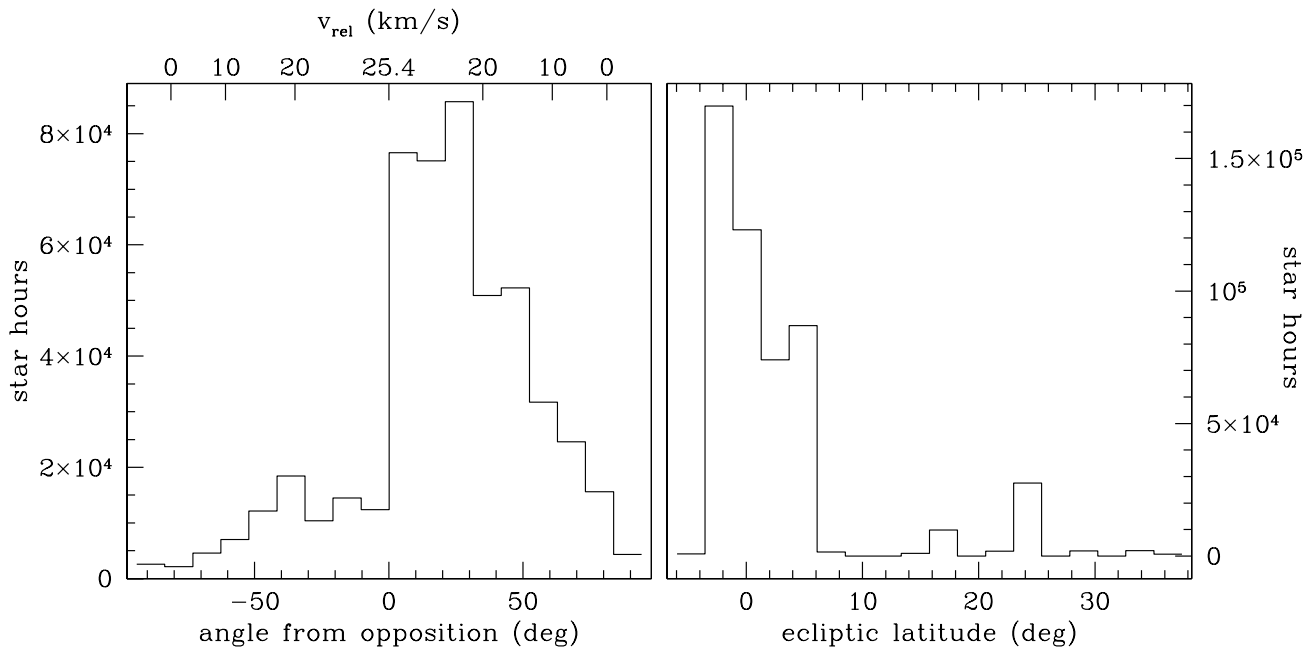

Figure 3. Left: distribution of angles from opposition for the TAOS targets. The top axis shows the relative velocity of a KBO at 43 AU, given the position of the field The bin size is 10.5. Right: distribution of ecliptic latitude for the TAOS target fields (center of the field is assumed), bin size $\sim 2.5$.
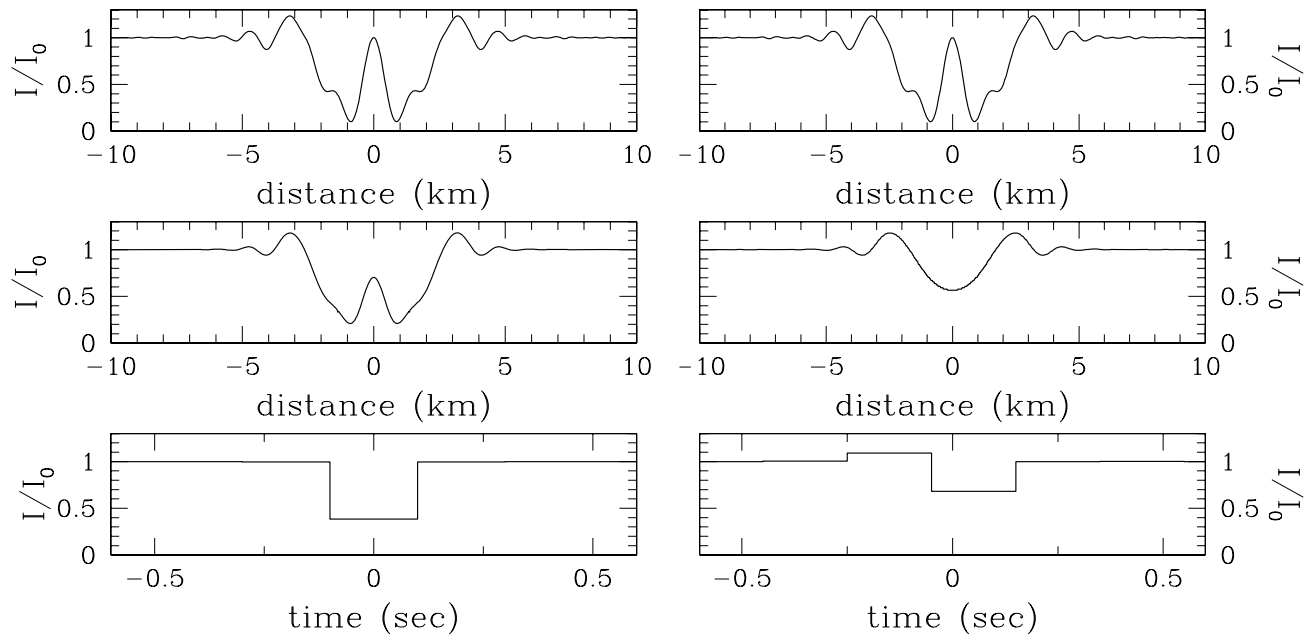

Figure 4. Steps of the generation of a simulated occultation event. Top, the left and right panels both show the same point source light curve for a $3 \mathrm{~km} \mathrm{KBO}$ at $43 \mathrm{AU}$ occulting an F0V star. Second row: finite source light curves for the same occultation parameters for a $V=11$ star, corresponding to an angular size of 0.015 mas, for a zero impact parameter (left), and at an impact parameter of $2 \mathrm{~km}$ (right). Row three: the light curves in row two are integrated over intervals of $105 \mathrm{~ms}$ for the occultation above at opposition (left) and at $50^{\circ}$ from opposition (right). The light curves are sampled at $5 \mathrm{~Hz}$, with no time offset (left) and with a time offset of $50 \mathrm{~ms}$ (right).

$0.2 \mathrm{~s}$ at opposition, and about a second near quadrature. A typical $\mathrm{KBO}$ occultation is then expected to result in the suppression of the flux for one or a few consecutive points in a TAOS light curve. In order to ascertain the extra-terrestrial origin of a dip in a light curve TAOS observes simultaneously with multiple telescopes. This allows us to rule out, on the basis of simple parallax considerations, atmospheric scintillation phenomena which might mimic an occultation event and which could be a source of false positives in occultation surveys, as well as any non-atmospheric phenomena such as birds, airplanes, etc.

In order to detect occultations we need to identify brief flux changes in a star simultaneously observed by all telescopes. The statistical significance of a simultaneous low point in our light curves can be assessed rigorously, and the probability of a low measurement being drawn out of pure noise decreases with the number of telescopes observing the target, provided that the measurements for the telescopes are independent. The light curves are high-pass filtered to remove trends due to weather patterns and changes in atmospheric transparency. High-pass filtering the light curves preserves the information on timescales relevant to occultation phenomena (one or a few points in a time series). The implementation of the filter is described in Z08. The filter produces a time series in which $h(t)$, the measurement taken at time $t$, represents the deviation from the local mean of the light curve in units of local standard deviation.

To detect events we rank-order the photometric measurement in each of our light curves, from the lowest to the highest flux, independently for each telescope (labeled A, B, and D). The ith point in a light curve will be associated with rank $r_{i}^{\mathrm{T}}$ for telescope $\mathrm{T}$. We then consider the rank triplets $\left(r_{i}^{\mathrm{A}}, r_{i}^{\mathrm{B}}, r_{i}^{\mathrm{D}}\right)$. The probability distribution of the quantity $z_{i}=-\ln \left\{r_{i}^{\mathrm{A}} r_{i}^{\mathrm{B}} r_{i}^{\mathrm{D}} / N_{\mathrm{p}}^{3}\right\}$, with $N_{\mathrm{p}}$ being the number of points in the light curve set, can be determined combinatorially. Knowing this, under the null hypothesis that there is no event in the triplet $i$, we can compute the probability for a random variable $Z$ arising from this distribution $P\left(Z>z_{i}\right)=\xi$. We set a threshold such that we expect fewer than 0.27 events in our data set that are due to random fluctuations. For the data set discussed in this paper we accept as events all data points that produce a rank product less likely than $\xi=3.0 \times 10^{-11}$ to be drawn from a random 
distribution. Note that events generated by large KBOs, or for observations near quadrature, would affect more than one point in the light curve (Figure 4), and our rank-based search algorithm is most efficient when the dip in the light curve is isolated. Therefore, in addition to searching for single-point events, we also bin our light curves by two, re-rank them and repeat the statistical tests described above. The probability of each data point is assessed for both unbinned and binned light curves. Each light curve is binned twice, with two different starting points. This increases the detectability of occultations by large KBOs and by KBOs transiting with low relative velocity. For a detailed discussion of our statistical analysis see Lehner et al. (2010).

For a set of light curves of a given star, the ranks in the three telescopes should not be correlated for the statistical analysis described above to be valid. We have developed a series of statistical tests to identify data runs where significant correlations (typically due to fast moving cirrus clouds) are found in the light curves. In such data runs the ranks are not independently distributed and thus we cannot accurately determine the statistical significance of any candidate events. Any data run where the independence of the measurements after filtering cannot be rigorously established is removed from our data set. A complete description of these tests is beyond the scope of this paper, but they are discussed in detail in Lehner et al. (2010).

For the next step, we considerably relax the ultimate significance requirement described above, and select as provisional candidates those triplets that have $\xi \leqslant 1.0 \times 10^{-6}$. Note that the significance $\xi$ refers to the probability that the point would be drawn from a random distribution, therefore the lower the value of $\xi$ the higher the statistical significance of the event. Nearly 150,000 provisional candidates are found. We use all these measurements to identify and remove spurious regions of the light curves, and hence identify and reject false positive events which arise from sources other than random chance. The constraints described below allows us to recognize regions of light curves with atypical noise and contamination by transiting objects (satellites or meteors, which turn out to be the major source of false alarms), and to identify high-frequency fluctuations in the raw data that are not removed by the high-pass filter. These are the steps of our false positive rejection process.

1. Contiguity: contiguous candidates within a light curve and candidates that are within three timestamps of each other are removed. Only the one rank triplet that has the highest significance in a series of contiguous or proximate points is considered as a candidate. This removes double-counted events: events caused by large KBOs or KBOs moving at low relative velocity would affect more than one contiguous point. Furthermore, this removes events that are doublecounted because they appear significant in both the binned and unbinned light curves. This eliminates about $40 \%$ of the candidates.

2. Simultaneity: candidates that appear in the light curves of more than one star simultaneously at the same time-stamp or within three rowblocks are considered to be false positives. We expect simultaneous count drops in time domain to be primarily due to inaccurate aperture positioning in the photometry. In the rowblock domain, simultaneous count drops might be due to inaccurate background determination or non-occultation events altering the baseline of the light curve at, or around, the candidate event, or by fast moving cirrus clouds or other phenomena which induce high frequency fluctuations in multiple light curves which are not removed by the high-pass filter. This cut removes about $60 \%$ of the remaining candidates.

3. Number of telescopes: at this point, we require all of our remaining candidates to have been observed by all three telescopes. Although we are only considering threetelescope runs in our analysis, for some targets the light curve might not be extracted in the photometry phase for all telescopes. Small differences in the field of view and in the field distortion might make one star target not visible to all telescopes if it is at the edge of the field or if the crowding induced by the zipper-mode readout caused overlap of the target with other stars (Lehner et al. 2009). About 35\% of the remaining candidates are thus removed. Note that this cut cannot be applied earlier as simultaneous events might appear in only one three-telescope light curve, but also in two-telescope light curves, and we want to be able to recognize and remove these events. At this point there are still over 20,000 candidates left. Note that we do not count the star hours discarded by this cut in our total exposure of $5 \times 10^{5}$ star hours.

4. Significance threshold: we finally constrain $\xi$ such as to expect fewer than 0.27 false positives in our data set. This constraint depends on the size of the data set: for the $9 \times 10^{9}$ triplets remaining $\xi<3.0 \times 10^{-11}$ allows $<0.27$ false positives due to random noise. Only 228 candidates remain.

The remaining candidates require visual inspection: first of the light curves, and for any remaining candidates, of the images. Most of the events are caused by the passing of bright objects, such as artificial satellites, meteorites or asteroids, that generate a variation in the background or baseline of the light curve responsible for causing artificially low counts in the neighborhood of the object. Many, but not all, of these false positives are removed by the simultaneity cut described above. Note that in our observing mode bright stars generate a bright streak across the length of our images (see Zhang et al. 2009), as flux is collected during the shutterless row shifting. In the presence of a bright object overlapping with a star streak generated by the zipper-mode readout, the brightness of the streak is overestimated, thus too much flux is subtracted from the rowblock column causing an artificial flux drop in the star time series. In many instances, the foreground object will also appear inside the star aperture artificially boosting its brightness. This flux drop will then be associated with a very high flux measurement following or preceding the event epoch, a signature that allows us to remove these false positives by inspecting the light curve. We also inspect the centroid position of the aperture. If the aperture position has moved significantly at the time stamp of the candidate the candidate is rejected. Of the remaining candidates, $90 \%$ are rejected by visual inspection of the time series.

Finally, we inspect the images of the remaining 23 candidates: they also were all associated with bright moving objects overlapping star streaks. No candidate events were left in our data set at the conclusion of this process.

\subsection{Determination of the Stellar Angular Size}

The shape of a light curve during an occultation event, and hence the detection efficiency, is strongly dependent on the angular size of the target star (Roques \& Moncuquet 2000; Nihei et al. 2007; Bickerton et al. 2009). Our fields contain a variety of stellar types and a large range of angular sizes (Figure 5(a)). 

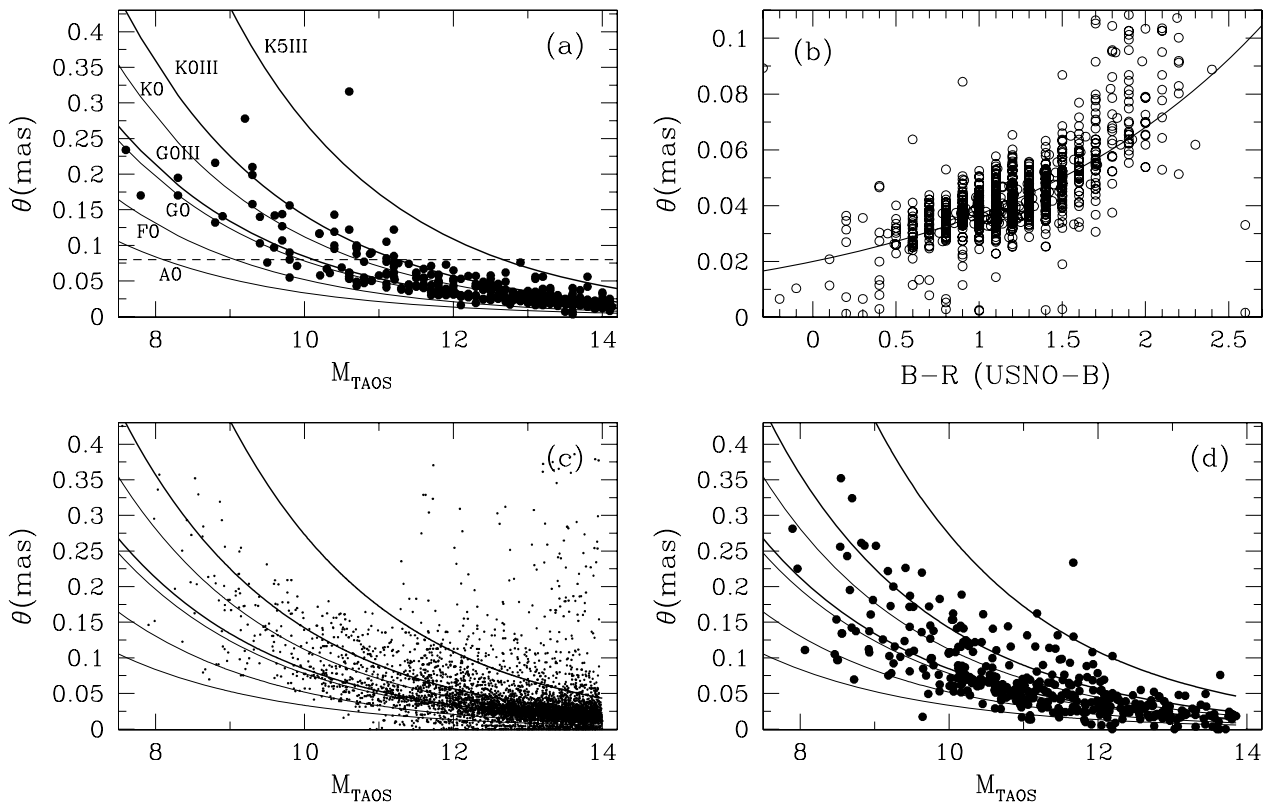

Figure 5. Angular size distribution for a typical TAOS field (field 120, R.A.: 13:7, Dec: -10.7 ) derived from the 2 MASS $K-J$ colors (a). The curves show the theoretical behavior of the angular size for A0, F0, G0, K0 dwarf stars (thin lines) and G0, K0, K5 giants (thick lines). The size of the Fresnel scale at 43 AU is shown at 0.08 mas (dashed line). Best fit to the angular size distribution (b): $x$-axis is the USNO-B $B-R$ color and $y$ is the angular size derived using Equations (1) and (2), converting all stars to apparent magnitude $R=12$. Implanted angular sizes, derived from USNO-B the $B-R$ color for our simulation (c) and angular size of the star for which events are recovered (d); a random subset of $1 \%$ of our data is plotted in the bottom panels.

Here, we describe the method we use to estimate the angular sizes of our target stars in order to account for this effect when estimating our detection efficiencies (see Section 3.3).

Angular sizes have been related to the position of a star in the color-color or color-magnitude diagrams (e.g., van Belle 1999; Nordgren et al. 2002). We follow the work of Nordgren et al. (2002) and calculate the angular size of our star targets using the Two-Micron All-Sky Survey (2MASS) $J$ and $K$ color (Cutri et al. 2003) to invert the set of equations:

$$
\begin{aligned}
& F_{K}=(3.942 \pm 0.006)-(0.095 \pm 0.007)(J-K), \\
& F_{K}=4.2207-0.1 K-0.5 \log \theta,
\end{aligned}
$$

where $F_{K}$ is the surface brightness of a star in $K$ band, which is related to its $J-K$ color, as well as to its unreddened apparent $K$ magnitude and angular size $\theta$. The relationship between the surface brightness and the color of a star (Equation (1)) is calibrated using angular sizes measured directly by long baseline interferometry (Nordgren et al. 2002).

Not all of our target stars, however, are identified 2MASS objects, while in the photometry phase we have identified all of our targets with USNO-B objects. We therefore devised a method that relies on USNO-B $R$ and $B$ magnitude to calculate the angular sizes of our targets.

We first derive the angular size of a subset of targets identified with 2MASS objects using the above equations, and scale it to obtain the angular size the targets would have if their apparent magnitude were $R=12$. We then considered the USNO-B $B-R$ color for all of these targets and calculated a regression on these points. This generates a formula that allows us to go from the USNO-B color of any of our targets to 2MASS colors and thus predict angular sizes according to Equations (1) and (2), for an apparent magnitude $R=12$. To calculate the true angular size we rescale from $R=12$ to $R_{\mathrm{USNO}} .{ }^{23}$ The angular sizes

\footnotetext{
${ }^{23}$ We do not use our instrumental magnitude for rescaling for consistency
} with what is used in the color determination. of a subset of TAOS targets, rescaled to $R=12$, is plotted as derived from Equations (1) and (2) versus the USNO-B $B-R$ color (Figure 5(b)). Our regression on the data is plotted as well (solid line).

The scatter in the determination of the angular size via the method described above is large, as can be seen in Figure 5(b). This is due to scatter in the USNO-B color $(\approx 0.3 \mathrm{mag}$; Monet et al. 2003), to the (much smaller) scatter in the $J$ and $K$ magnitudes, and to the scatter in the empirical determination of the relationship between $\theta$ and $J-K$ in Equations (1) and (2). We have not used any interstellar reddening corrections, and the angular size estimation of an unknown reddened star from the near-IR relationship would be relatively less affected compared to that in visual bands. Reddening is typically small for our targets though, since we are only considering objects brighter than $R \sim 13.5$.

The distribution of angular sizes is well reproduced. Figure 5(a) shows the distribution of angular sizes for a typical TAOS field, calculated via Equations (1) and (2), and Figure 5(c) shows the distribution of angular sizes in our efficiency simulation obtained via the USNO-B color. The distributions do overlap. About $2 \%$ of our simulated angular sizes fall in the region $\theta>0.15$ mas and $R_{\mathrm{TAOS}}>11$, where there are no observed objects. These objects have poor USNO-B color determination. Figure 5(d) shows the region of the $\theta-R_{\text {TAOS }}$ space where simulated events are recovered. There are few recoveries in the region $\theta>0.15$ mas and $R_{\text {TAOS }}>11$, so these stars do not contribute the expected event rate.

\subsection{Detection Efficiency}

It is necessary to assess the efficiency of our recovery algorithm in order to derive the number density of KBOs from the number of events in our survey. In order to measure our recovery efficiency we implant our data with synthetic occultations. The data are then reprocessed in the same way we did to search for true events. By implanting into the 
Table 2

Distribution of Synthetic Events

\begin{tabular}{lcc}
\hline \hline Diameter $(\mathrm{km})$ & Implantations & Recoveries \\
\hline 30.0 & 231 & 75 \\
8.0 & 385 & 84 \\
3.0 & 1078 & 73 \\
2.0 & 2003 & 89 \\
1.3 & 4393 & 73 \\
1.0 & 13255 & 66 \\
0.7 & 36222 & 40 \\
0.5 & 447764 & 9 \\
\hline
\end{tabular}

actual light curves we do not make any assumption regarding the nature of the noise in our data. Note that our detection algorithm, described in Section 3.1, is not affected by the spectral characteristics of the noise, as long as the distribution of flux measurements in a light curve is stationary (Lehner et al. 2010). Our occultation simulator is based on the work described in Nihei et al. (2007).

We first generate diffraction light curves for KBOs occulting point sources. We integrate the diffraction pattern over the disk of our target star. Keeping the stellar type fixed, the angular size is modulated by changing the apparent magnitude of the star and we can use the point source light curve to integrate the occultation signature over the star disk. A point source light curve for a $D=3 \mathrm{~km} \mathrm{KBO}$ at $\Delta=43 \mathrm{AU}$ is shown in Figure 4, top, and the finite source light curve for a magnitude $V=11$ star is shown in the second panel, left. Note the smoothing of the diffraction features. We modify the light curve to account for a finite impact parameter $b$ by using the finite source light curve at impact parameter $b=0$ as an input and calculating the intensity of the occultation signal at the new distance of each point form the center of the diffraction pattern by interpolating points of the finite source light curve. A light curve for an F0V, $V=11$ star and a $3 \mathrm{~km} \mathrm{KBO}$ occulting at an impact parameter $b=2 \mathrm{~km}$ is plotted on the right-hand side of the second panel of Figure 4. Finally, after calculating the relative velocity of the $\mathrm{KBO}$ as a function of distance and angle from opposition as per Liang et al. (2004), and Nihei et al. (2007), we smooth the light curve to account for finite exposure intervals, and we sample the finite exposure light curve at the appropriate sampling rate. In this step we can account for dead time in the sampling interval, which for TAOS is $47.5 \%$ at $5 \mathrm{~Hz}$. We also allow an offset in time between the center of the finite sampled light curve and the integration bin. In the bottom row of Figure 4, the light curves for the event in row two are integrated over $105 \mathrm{~ms}$ second intervals and sampled at $5 \mathrm{~Hz}$, the typical sampling rate of TAOS, for an event at opposition and with no time offset (left), and for an event at $50^{\circ}$ from opposition with an offset of $50 \mathrm{~ms}$ between the center of the sampling interval and the center of the occultation (right).

In order to sample properly the space of diameters to which the survey is sensitive we implant synthetic occultations by objects of diameter $D=0.5,0.7,1.0,1.3,2.0,3.0,8.0$, and $30.0 \mathrm{~km}$. For a $30 \mathrm{~km}$ diameter KBO the event falls in the geometric regime; diffraction effects are therefore no longer significant and our efficiency stabilizes. Because our sensitivity decreases with decreasing diameter we implant progressively more objects at smaller diameters. The number of implantations at each size is designed to allow us to obtain a good sampling at all sizes. In Table 2, we report the number of objects implanted for each size in one of our efficiency runs and the number
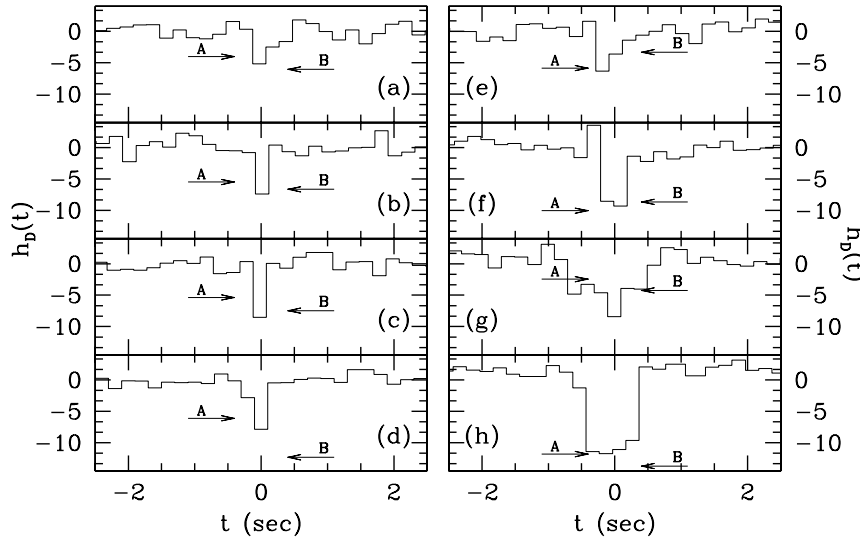

Figure 6. Implanted occultations recovered by our pipeline. On the left the light curves refer to star targets in a field observed near opposition and on the right in a field observed at large angle from opposition. $h(D)$ represents the deviation at that epoch from the local mean of the light curve in units of standard deviation as measured by telescope TAOS D. The values of $h_{\mathrm{A}}(0)$ and $h_{\mathrm{B}}(0)$, for TAOS A and $\mathrm{B}$, are indicated by horizontal arrows labeled $\mathrm{A}$ and $\mathrm{B}$, respectively. Each event is described in Table 3.

of recoveries. ${ }^{24}$ For objects within the Kuiper Belt (about 30-60 AU), the differences induced by different distances are negligible in the occultation features as observed by TAOS. We therefore set the distance to $\Delta=43$ AU. Every occultation event is implanted at a random epoch in the light curve set and at a random impact parameter between 0 and $H / 2$, where we set $H$, a measure of the cross section of the event, to the size of the Airy ring and the projected size of the star in accordance to Nihei et al. (2007).

In order to implant the synthetic occultations into our data we modulate the light curve by subtracting (adding) the amount of flux suppressed (augmented) by the occultation at each datapoint as done in Z08. This approach slightly overestimates the noise due to Poisson statistics where the flux is suppressed, giving us a conservative estimate of our efficiency. We implant exactly one occultation in each light curve in our data set.

We then process our implanted light curves as we previously did to search for events, namely we filter, rank the light curves, and evaluate the significance $\xi$ of each point in each light curve. We then remove the false positives as described in Section 3.1. Our efficiency decreases rapidly with the KBO diameter: from nearly $33 \%$ at $D=30 \mathrm{~km}$ to $2 \times 10^{-5}$ at $D=0.5 \mathrm{~km}$. Note that at $D=30 \mathrm{~km}$ we ignore diffraction effects and the occultations are modeled to suppress the flux completely for several consecutive points, depending on the relative velocity, but our efficiency is still significantly less than $100 \%$. Some of our light curves are too noisy to allow detections.

A set of synthetic events recovered by our pipeline is shown in Figure 6, and the parameters of each plotted event are given in Table 3. Various parameters affect our recovery efficiency. Our efficiency for the recovery of $D=3.0 \mathrm{~km}$ occulting KBOs is plotted in Figure 7.

The efficiency as a function of $\mathrm{S} / \mathrm{N}$ is plotted in Figure 7(a)a few targets at $\mathrm{S} / \mathrm{N}>100$ are left out of the plot. The behavior of our efficiency as a function of crowdedness, where the crowdedness is defined as the number of targets in that TAOS field brighter than $R_{\mathrm{USNO}}=13.5$, is plotted in Figure $7(\mathrm{~b})$. The efficiency is here plotted multiplied by the number of targets in the field, to give a better idea of the implication of this parameter

\footnotetext{
24 Four runs are conducted to improve statistical accuracy in the determination of our efficiency.
} 

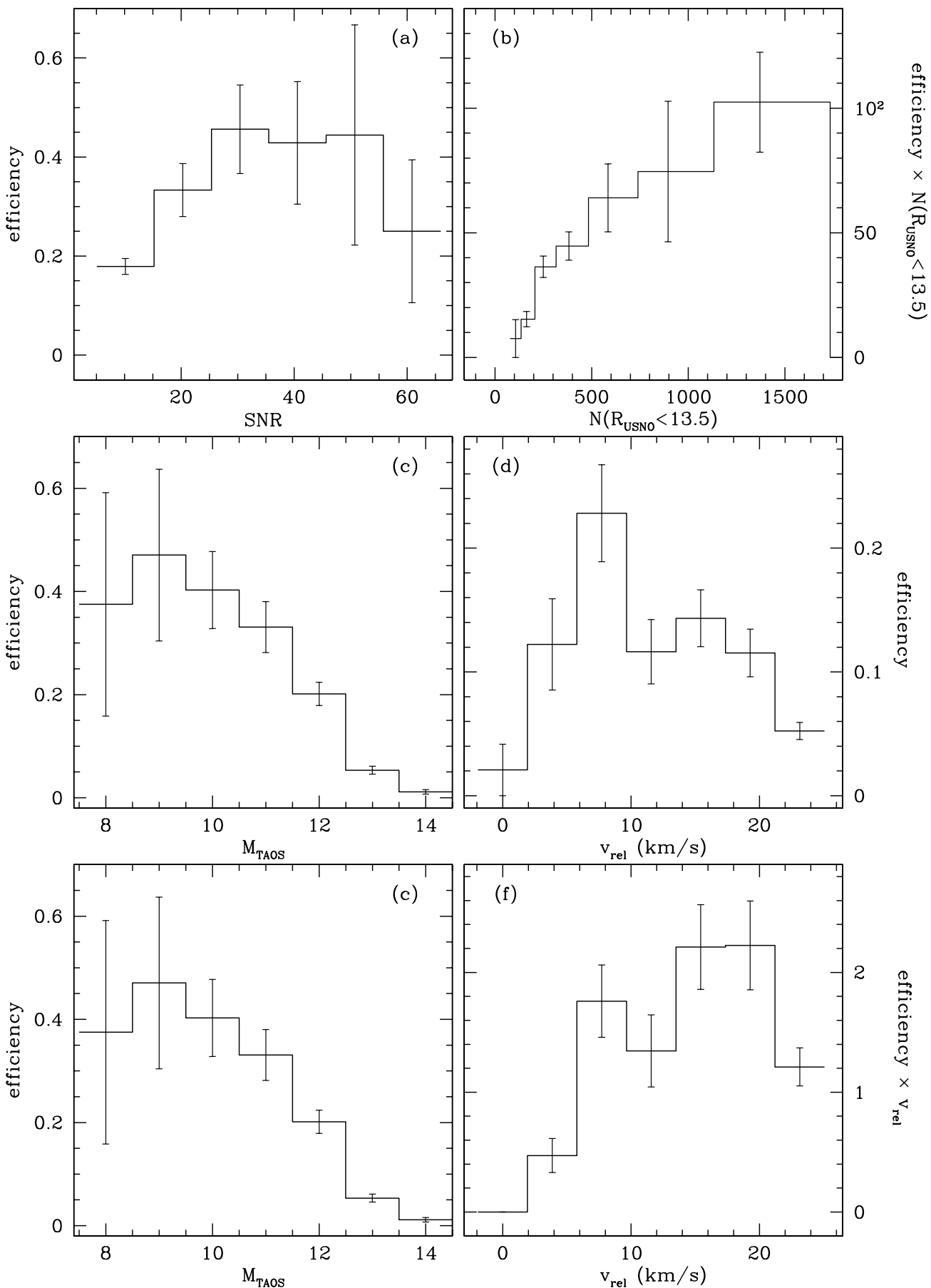

Figure 7. Recovery efficiency for $3 \mathrm{~km} \mathrm{KBOs.} \mathrm{Panel} \mathrm{(a):} \mathrm{efficiency} \mathrm{as} \mathrm{a} \mathrm{function} \mathrm{of} \mathrm{S/N.} \mathrm{Efficiency} \mathrm{vs.} \mathrm{crowdedness} \mathrm{of} \mathrm{the} \mathrm{field} \mathrm{in} \mathrm{panel} \mathrm{(b),} \mathrm{defined} \mathrm{as} \mathrm{the} \mathrm{number}$ of targets brighter than $R_{\mathrm{USNO}}=13.5$. The efficiency is weighted by the crowdedness. Panel (c): efficiency vs. magnitude $M_{\mathrm{TAOS}}$. In panel (e), the efficiency as a function of magnitude is weighted by the number of targets at that magnitude. Panel (d): efficiency vs. relative velocity of the KBO targets. In panel (f), the efficiency vs. relative velocity is weighted by the relative velocity. All error bars are calculated in a Poissonian fashion from the square root of the number of recoveries.

Table 3

Parameter of Implanted Events in Figure 6

\begin{tabular}{lcccccrrr}
\hline \hline Figure Panel & $D(\mathrm{~km})$ & $b(\mathrm{~km})$ & $v_{\text {rel }}\left(\mathrm{km} \mathrm{s}^{-1}\right)$ & $\mathrm{S} / \mathrm{N}$ & $\theta_{\star}(\mathrm{mas})$ & $h_{\mathrm{A}}(0)$ & $h_{\mathrm{B}}(0)$ & $h_{\mathrm{D}}(0)$ \\
\hline (a) & 0.7 & 0.50 & 25.4 & 39.8 & 0.03 & -4.0 & -6.1 & -5.2 \\
(b) & 1.0 & 0.50 & 14.9 & 29.4 & 0.03 & -5.5 & -6.6 & -7.4 \\
(c) & 3.0 & 1.19 & 25.4 & 12.8 & 0.03 & -5.4 & -7.5 & -8.5 \\
(d) & 8.0 & 2.7 & 25.4 & 9.4 & 0.006 & -6.1 & -12.3 & -7.9 \\
(e) & 0.7 & 0.22 & 8.2 & 10.2 & 0.03 & -5.8 & -3.3 & -6.3 \\
(f) & 1.0 & 0.94 & 7.7 & 10.8 & 0.03 & -10.0 & -8.7 & -8.6 \\
(g) & 3.0 & 1.18 & 3.1 & 12.9 & 0.006 & -2.5 & -4.2 & -8.4 \\
(h) & 8.0 & 2.93 & 8.1 & 15.7 & 0.04 & -11.8 & -13.7 & -11.7 \\
\hline
\end{tabular}




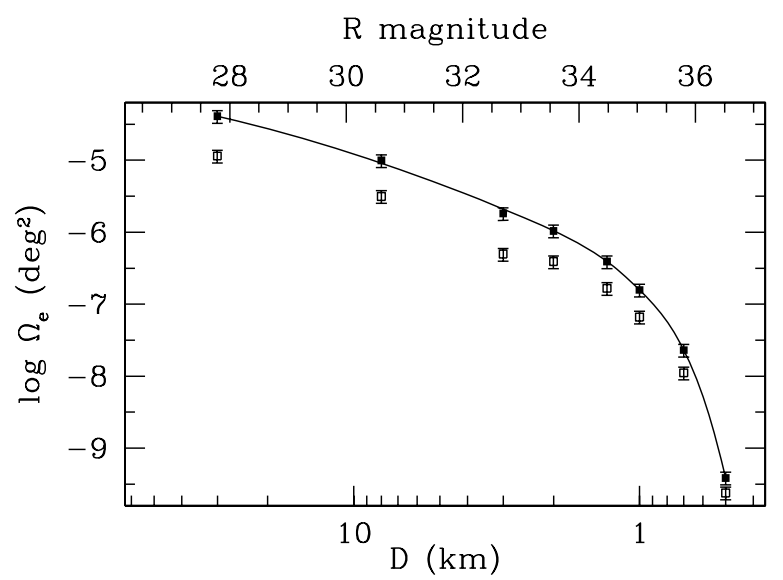

Figure 8. Effective solid angle for the first two years of TAOS data (Z08, empty squares) and for the current 3.75 year data set (solid line and filled squares).

for the event recovery. The largest number of detections is achieved for more crowded fields.

The efficiency decreases with magnitude (Figure 7(c)) by about a factor of 5 between magnitudes 9 and 13. The dominant effect here is the decrease in $\mathrm{S} / \mathrm{N}$, though a competing effect occurs since lower magnitudes are associated with larger angular sizes, and our efficiency decreases with increasing angular size (Figure 5). Furthermore, there are many more dim than bright stars in the sky: Figure 7(e) shows the efficiency as a function of $M_{\text {TAOS }}$ multiplied by the number of TAOS targets at that magnitude. The highest number of detections happens for stars with $M_{\text {TAOS }} \sim 12.5$.

Our efficiency as a function of the relative velocity of the $\mathrm{KBO}$ is plotted in Figure 7(d). Observing at a pointing where the relative velocity of the KBOs is higher boosts the event rate of the survey. Our efficiency, however, is larger for smaller transiting velocities, particularly for small KBOs for which the time line of the event is shorter than one of our datapoints at opposition. Ultimately, the effective sky coverage of our survey depends linearly on both the efficiency and the velocity (see Section 3.4). The efficiency multiplied by the relative velocity $v_{\text {rel }}$ is plotted against $v_{\text {rel }}$ in Figure 7(f). Pointing near opposition increases the effective coverage of our survey, and thus it increases our event rate, for $3 \mathrm{~km}$ KBOs. The survey strategy can be optimized at different sizes taking into account the size-dependent efficiency as well as the expected KBO size distribution.

\subsection{Effective Sky Coverage and Upper Limits}

We calculate the effective sky coverage of our survey, $\Omega_{\mathrm{e}}$, as

$$
\Omega_{\mathrm{e}(\mathrm{D})}=\frac{1}{w(D)} \sum_{*} \frac{H\left(D, \theta_{*}\right)}{\Delta} \frac{v_{\mathrm{rel}}}{\Delta} E_{*}
$$

where $E_{*}$ is the exposure of the star target (the duration of the light curve set), $\Delta$ is the distance to the occulter, $H$ is the cross section of the event (Section 3.3), w(D) is the weight factor for that diameter, i.e., the fraction of light curves implanted with occultations by KBOs of diameter $D$, and the sum is carried out only over the light curves where events are recovered.

The effective coverage of our survey, which takes into account our efficiency, is plotted in Figure 8, for both the data set published in Z08 (empty squares) and for the current work (filled squares). The solid line is a spline fit to the points.

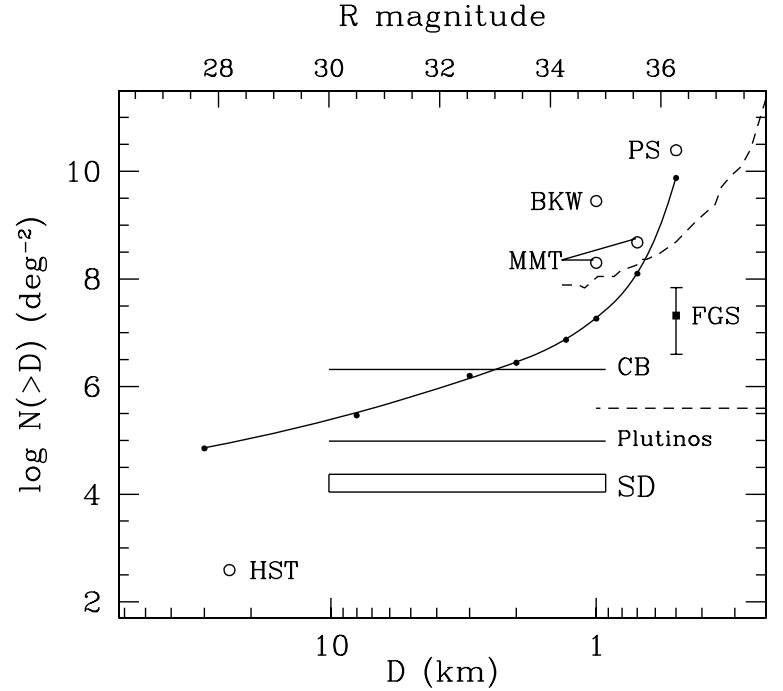

Figure 9. Model-independent upper limits from the TAOS survey (solid dots and solid line). Each point represents the upper limit to the number of $\mathrm{KBO}$ of that size or larger given the TAOS effective coverage. Similarly derived upper limits from Bickerton et al. (2008) at $1 \mathrm{~km}$ (BKW), Wang et al. (2009) at $0.5 \mathrm{~km}$ (PS) and Bianco et al. (2009) at $1 \mathrm{~km}$ and $0.7 \mathrm{~km}$ (MMT) are plotted as empty circles. The upper limit from Bernstein et al. (2004, direct survey) is also plotted (HST). The dashed lines represent the 95\% upper and lower limits from S09. The black square (FGS) represents the best-fit density for the entire S09 survey, with $1 \sigma$ error bars. The estimates of the number of objects in the $\mathrm{CB}$, of Plutinos, and SD objects are plotted, as derived by Levison \& Duncan (1997), Morbidelli (1997), and Volk \& Malhotra (2008) respectively, assuming each family is the unique precursor of JFCs.

\section{MODEL-INDEPENDENT LIMITS ON THE SIZE DISTRIBUTION OF KBOs}

We can use $\Omega_{\mathrm{e}}$ to calculate model-independent upper limits: at each size for which our efficiency calculation was conducted we calculate the number density of KBOs from the TAOS data set as a single-point upper limit. These limits are shown as filled dots. We can then interpolate these upper limits with a spline fit, obtaining the solid line in Figure 9. At each diameter $D$ this represents the maximum surface density of KBOs of diameter $\gtrsim D$. Figure 9 shows model-independent upper limits reported by the occultation surveys of Bickerton et al. (2008), Bianco et al. (2009), and Wang et al. (2009). The improvement is of nearly an order of magnitude at $700 \mathrm{~m}$ and over an order of magnitude at $1 \mathrm{~km}$ from the results of Bianco et al. (2009) and at $500 \mathrm{~m}$ from Wang et al. (2009). As discussed in Sections 1 and 3, the TAOS sensitivity is limited to relatively bright stars $(V \lesssim 13.5)$, and TAOS has a relatively low efficiency at detecting $D<3 \mathrm{~km}$ KBOs. The large star exposure of TAOS, however, provides a data set that well compensates for the lower sensitivity.

Limits from the recent $H S T / \mathrm{FGS}$ occultation survey (Schlichting et al. 2009, hereinafter S09) are included in Figure 9. S09 reported the detection of one possible event at a relatively high ecliptic latitude. The event is consistent with an occultation by a $\mathrm{KBO}$ with $D=1.0 \mathrm{~km}$. The dashed lines in Figure 9 represent the $95 \%$ confidence level (c.l.) upper and lower limits from S09. The TAOS upper limits are more constraining than the corresponding limits from S09 for $D \gtrsim 0.6 \mathrm{~km}$. The black square (FGS) represents the best-fit density for the entire S09 survey, with $1 \sigma$ error bars. Note that this is not a model-independent estimate, as it is derived assuming a straight power-law distribution for small KBOs. Note also that TAOS has a greater sky coverage at $D \sim 1 \mathrm{~km}$, where the S09 event 


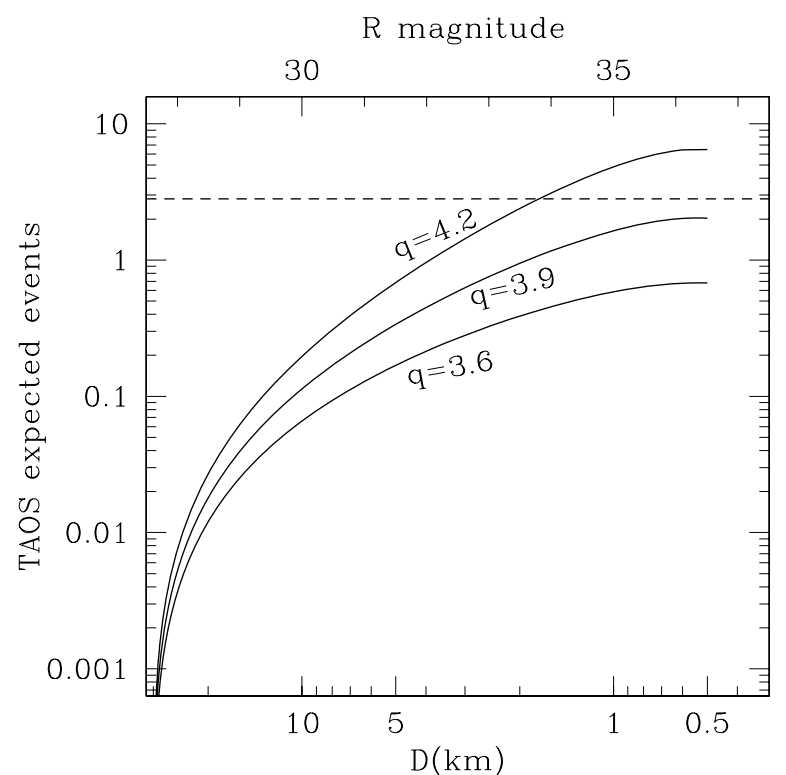

Figure 10. Expected number of events in 3.75 years of TAOS data from the size distribution presented in S09. Slopes of $q=3.9$ (best fit to the HST/FGS data), $q=3.6$ and 4.2 ( $\pm 1 \sigma$ from the best fit) are used.

was detected, by a factor of $\sim 6$. This detection reported by S09 is, however, not statistically inconsistent with our upper limit.

Figure 10 shows the expected number of events for the TAOS survey if one assumes the size distributions derived in S09. S09 estimated a cumulative size distribution $\Sigma_{N}(D>0.5 \mathrm{~km})=2.1 \times 10^{7} \mathrm{deg}^{-2}$ from their detection and non-detections, and fit a power law to their data, anchored at $\Sigma_{N}(D>90 \mathrm{~km})=5.4 \mathrm{deg}^{-2}$, to yield a differential slope of $q=3.9 \pm 0.3$. Figure 10 shows the expected number of events for the TAOS survey for these distributions. We calculate the number of events expected in our survey as

$$
N_{\exp }=\int \frac{d N}{d D} \Omega_{\mathrm{e}} d D .
$$

With no detections our survey can rule out distributions that predict $N_{\exp } \geqslant 3$ at the $95 \%$ c.l.. The best-fit S09 model leads to an expected number of 2.1 detections in our TAOS data. This is a reasonable consistency. We can rule out slopes steeper than $q=4.0$ for this model of the size distribution at $95 \%$ c.l.

Figure 9 also shows the estimates of JFC progenitor populations. Our constraints on these families are discussed in Section6.

\section{OUTER SOLAR SYSTEM COLLISIONAL MODELS}

The collisional and dynamical evolution of the solar system shaped the size distribution of the Kuiper Belt.

The belt was originally populated by very small dust grains, with small orbital eccentricities $(e \leqslant 0.01)$ such as those we observe in circumstellar disks around other stars (MoroMartin et al. 2007). Initially these small objects merge and grow (Kenyon \& Luu 1999a): 1 km KBOs in the Kuiper Belt are thus formed. As their gravitational cross section grows larger than their geometric cross section, gravitational focusing speeds up the growth rate of the largest bodies. This phase is referred to as runaway growth, and objects as large as hundreds of kilometers can form. One such population, shaped primarily through agglomeration processes is predicted by theory to have a power-law distribution in diameter $d N / d D \propto D^{-q_{\mathrm{L}}}$ with power $q_{\mathrm{L}} \approx 4.5$ (Kenyon \& Luu 1999b). Direct observations of large KBOs confirm the power-law behavior in this regime, the gravitationally dominated region of the size spectrum, with a best fit of $q_{\mathrm{L}}=4.8$ (Fraser \& Kavelaars 2009; Fuentes \& Holman 2008, and reference therein). The size distribution of these large objects, for which gravity dominates the internal strength, is remarkably insensitive to parameters such as Neptune stirring or the internal tensile strength of the KBOs.

Meanwhile, very large objects in the planetary region of the solar system are also forming into planets, that are believed to undergo significant migrations (Tsiganis et al. 2005, and references therein). The orbits of the planetesimals are then stirred up via gravitational interaction to velocities such that further impacts will result in the disruption of the smaller objects: this is the catastrophic collisions phase (Davis \& Farinella 1997; Kenyon \& Luu 1999a; Morbidelli et al. 2008); the timescale to reach this phase is estimated to be between $10 \mathrm{Myr}$ and $1 \mathrm{Gyr}$ (Kenyon \& Bromley 2001). For very small objects (probably tens of meters and smaller) the collisionally evolved population transitions to a regime where the KBO binding energy is dominated by internal strength, rather than gravity. Here the collisional cascade will generate a size distribution which follows a power law with index $q_{\mathrm{S}}=3.5$ (Dohnanyi 1969; Kenyon \& Luu 1999a), also in a fashion that is largely independent on the details of the evolution of the protoplanets. Note that the study of collisions between icy bodies is still in its infancy, and future work in this field will permit assessing the behavior of colliding small strengthless or loosely bound particles (Leinhardt 2008). Similarly, future work on coupling collisional and dynamical evolution codes, recently pioneered by Charnoz \& Morbidelli (2007), should provide further insight in the behavior of the size distribution.

We will refer to the region in between these two regimes as the intermediate region. The extent of, and behavior of the size distribution in the intermediate region are instead very sensitive to the formation and evolution parameters, and observational information on this region can be compared to evolution models. In general, for a weaker KBO population the transition to the $q_{\mathrm{S}}=3.5$ power-law behavior will occur earlier, reducing the size of the transition region. A strong KBO population will display an extended intermediate region, generally showing here oscillations around a mean power law of slope which also depends on the details of the population and its evolution. In this section, we present four models, from the literature, of the KBO size distribution $d N / d D$, and a simple parametric model. On the basis of these models we calculate the number $N_{\text {exp }}$ of events expected to be detected by the TAOS survey. Since no events were found in our survey, any model which predicts $N_{\exp } \geqslant 3$ is ruled out by TAOS at the $95 \%$ c.l.

We caution the reader that the predictions presented here depend on both the shape of the size distribution and on scaling parameters. All of our models are scaled so that the differential size distribution is consistent with the direct observations at $D=200 \mathrm{~km}$, where the direct surveys are most constraining:

$$
d N / d D(D=200 \mathrm{~km}) \sim 0.04 \mathrm{deg}^{-2} \mathrm{~km}^{-1} .
$$

For each model we will point out the cumulative number of KBOs predicted at $D=100 \mathrm{~km}, \Sigma_{N}(D>100 \mathrm{~km})$, with this scaling choice. Strengths and weaknesses of all of these models are also discussed in Fraser (2009). 

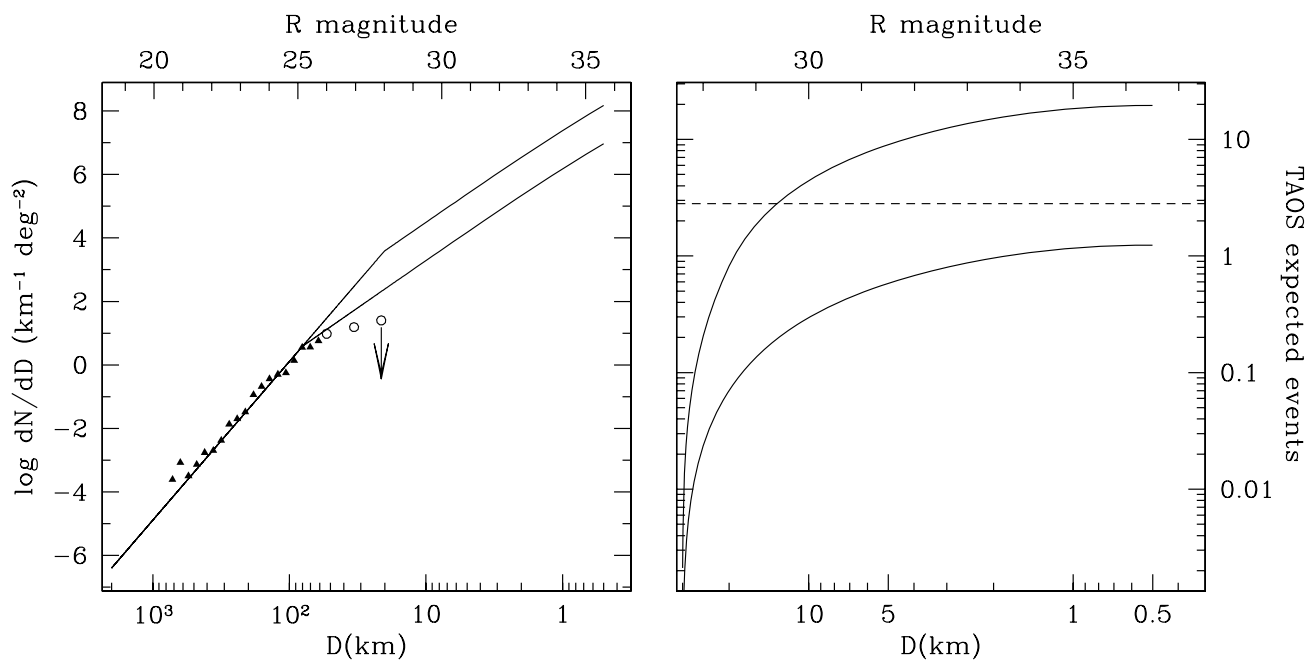

Figure 11. PS05 model and expected event rate for TAOS. Left: triangles are the data from Fraser \& Kavelaars (2009). Empty circles are the data from Bernstein et al. (2004). We use these data from direct imaging surveys to set the location of the large end size distribution. The model is parameterized with two slopes, $q_{\mathrm{L}}=5$ and $q_{\mathrm{I}}=3$. The positions of the break are plotted at $D_{\mathrm{b}}=80$, and $20 \mathrm{~km}$ : limiting values for the PS05 models. Right: expected yield of events in 3.75 years of TAOS data. The horizontal dashed line represents the highest number of events allowed given no detections in the TAOS data ( $\leqslant 3$ events at the $95 \%$ c.l.). Any model above this limit is ruled out by our survey.

\subsection{Pan \& Sari (2005)}

Pan \& Sari (2005, hereinafter PS05) derived a fully analytical model for the size distribution of KBOs by assuming the population is in a steady state and the mass is constant through the collisional processes. They assume for most of their model calculation that the internal strength of the objects is negligible (gravity dominated objects). This assumption is motivated by studies of comets and asteroids (PS05, and references therein). The transition to the fully strength-dominated regime, where the size distribution follows Dohnanyi (1969) with a power law with slope $q_{\mathrm{S}}=3.5$, occurs at $D \leqslant 300 \mathrm{~m}$. This region of the size spectrum is entirely below the sensitivity of TAOS.

PS05 derive an analytical double power-law size distribution for objects $D \geqslant 300 \mathrm{~m}$ :

$$
\begin{aligned}
& d N / d D \propto d^{-q_{\mathrm{L}}} \quad \text { for } \quad D>D_{\mathrm{b}}, \\
& d N / d D \propto d^{-q_{\mathrm{I}}} \quad \text { for } \quad D<D_{\mathrm{b}} .
\end{aligned}
$$

This model is shown in Figure 11, left. The slope $q$ has value $q_{\mathrm{L}}=5$ for large objects and $q_{\mathrm{I}}=3$ for objects in the intermediate region. PS05 are thus able to calculate self-consistently the location of the break in the power $D_{\mathrm{b}}$, which represents the size of the largest KBOs that experienced catastrophic collisions, as a function of time. The location of the break moves toward larger objects as the size distribution evolves.

Model distributions with break points $D_{\mathrm{b}}=80$, and $20 \mathrm{~km}$, limiting values for PS05, and $\Sigma_{N}(D \geqslant 100 \mathrm{~km}) \sim 32 \mathrm{deg}^{-2}$ are shown in Figure 11. The corresponding predicted number of events for the TAOS data analyzed in this paper is plotted in the right panel. ${ }^{25}$

The structure of the size distribution in the intermediate region is however generally more complicated. Between $D_{\mathrm{b}}$ and the second break, which is the region that TAOS can probe, a realistic size distribution is expected to have an oscillatory

\footnotetext{
${ }^{25}$ Note that a simple extension of the large-size power-law distribution, which represents the naive expectation before Bernstein et al. (2004) appeared, would lead to thousands of detections. This was the initial design target of the TAOS project.
}

behavior which in the PS05 models preserves an average slope of $q_{\mathrm{I}}=-3$ (PS05). These waves however, as they are described in PS05, are small in amplitude and affect our prediction to less than $1 \%$. On the basis of our no-detection result, break diameters of $D_{\mathrm{b}}<51.3 \mathrm{~km}$ are excluded at $95 \%$ c.l. This is consistent with the data from direct observations and with the authors' interpretation of the model: in the absence of stirring by Neptune, the location of the break is consistent with a KBO population comprised of objects with little internal strength. Note that direct surveys also suggest that for one such distribution the location of the break should be at a large diameters (see Fraser \& Kavelaars 2009 and Figure 11, left).

\subsection{Kenyon \& Bromley (2004)}

Kenyon \& Bromley (2004, hereinafter KB04) developed numerical models of the collisional evolution of $\mathrm{KBOs}$, including the effects of the internal strength and gravitational binding of KBOs, as well as the initial mass in the belt and a model of stirring by Neptune: if in its migration Neptune reaches its current location early its stirring effect will influence the shaping of the size distribution of the Kuiper Belt. The disruption energy, defined as the energy necessary to remove $50 \%$ of the combined mass of the colliding bodies, is modeled as $Q_{d}=Q_{b} r^{\beta_{b}}+\rho Q_{g} r^{\beta_{g}}$, where $Q_{b}$ and $\beta_{b}$ describe the internal binding energy and $Q_{g}$ and $\beta_{g}$ the gravitational energy, with $\rho$ being the density and $r$ the radius of the object. In the KB04 simulations $Q_{b}$ is varied between 10 and $10^{8} \mathrm{erg} \mathrm{g}^{-1}, \rho Q_{g}$ between $10^{-4}$ and $10^{4}$, and $\beta_{g}$ between 0.5 and 2.0, while $\beta_{b}$ is set to 0 , as this parameter has little effect on the simulation results.

All KB04 models generally agree in the shape and slope of the size distribution for large $\mathrm{KBOs}(D=80 \mathrm{~km}$ and larger) generating a cumulative size distribution which follows $N(D \gtrsim 80 \mathrm{~km}) \propto D^{3.5}$, equivalent to a power-law differential size distribution with power $q_{\mathrm{L}} \approx 4.5$, in good agreement with data and theoretical predictions for the evolution in the gravitationally dominated regime. The models display a variety of behaviors for smaller objects. In all simulations a small dip in the $10-40 \mathrm{~km}$ region is predicted (cf. Figures 10 and 11 in KB04). This is roughly consistent with the results of 
$R$ magnitude

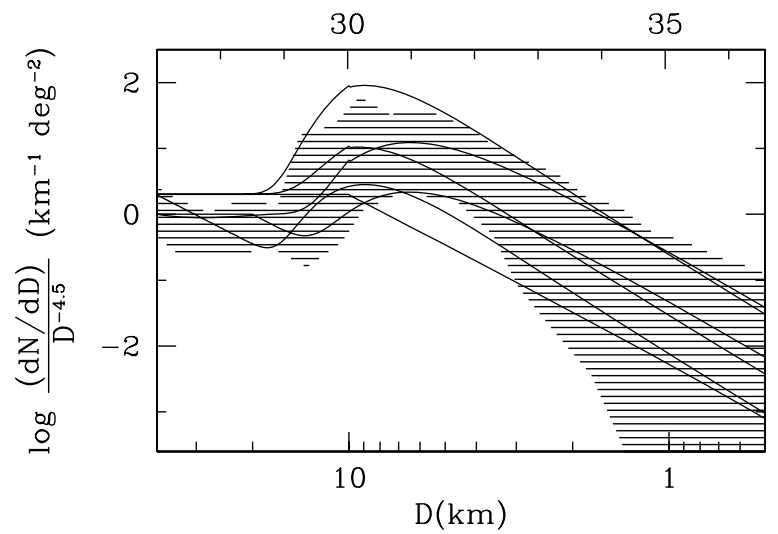

Figure 12. Our modeling of the KB04 results. The range of results of the simulations of KB04 is represented by the shaded region (see Figures 10 and 11 in KB04). A few of our models are plotted as solid lines. All models are normalized by the slope of the large KBO size distribution $D^{-4.5}$.

Bernstein et al. (2004): here collisions destroy weak KBOs and models with Neptune stirring or weakly bound KBOs produce a more significant dip. This feature is followed by an excess with respect to the nominal power law for $2-15 \mathrm{~km} \mathrm{KBOs}$. The amplitude of the excess varies substantially, between a factor of a few and a factor of a few tens, depending on the internal strength of the objects and on the details of the effect of Neptune stirring. The size distribution remains sensitive to the details of the models down to about $D=50 \mathrm{~m}$, where once again a power-law behavior begins, with power $3<q<5$ in the strength-dominated regime.

Figure 12 shows our representation of the models in KB04, as they are presented in Figures 10 and 11 of KB04. The size distribution is here shown scaled by the slope of the large size region $\left(D^{-4.5}\right)$. The shaded region represents the range of the KB04 models and the plotted lines are some of our models, covering a large region of this model space. Focusing on the region near the transition between primordial and collisionally evolved population, an excess near $5 \mathrm{~km}$ and a depletion near $25 \mathrm{~km}$ are both visible and well represented in our models.

We model the large size distribution as a power law with $q_{\mathrm{L}}=4.5$ and the intermediate region with $q_{\mathrm{I}}=2$. We model the excess near $D=5 \mathrm{~km}$ with a Gaussian, so that

$$
\frac{d N_{\mathrm{ex}}}{d D}=\frac{d N}{d D}\left(1+I_{\mathrm{ex}} \exp -\frac{\left(D-\mu_{\mathrm{ex}}\right)^{2}}{2.0 \sigma_{e}}\right) .
$$

We fix the width of the Gaussian excess to $\sigma_{e}=3.5 \mathrm{~km}$, we set the location to $\mu_{\mathrm{ex}}=5.5 \mathrm{~km}$ or $\mu_{\mathrm{ex}}=1.6 \mathrm{~km}$. The intensity of the excess is determined by $I_{\mathrm{ex}}$; in order to fairly represent all results from the KB04 simulations we consider models with an excess of $I_{\mathrm{ex}}=0,10$, and 100 . Note that as the break diameter moves toward large sizes the models naturally simulate the small dip near $20 \mathrm{~km}$ (Figure 12). Our results are not sensitive to the presence of this small depletion. With our scaling we obtain a cumulative surface density of KBOs $N(D \geqslant 100 \mathrm{~km}) \sim 25 \mathrm{deg}^{-2}$.

Figure 13 shows the models with $\mu_{\mathrm{ex}}=5.5 \mathrm{~km}$ (left) and the corresponding expected number of events for the TAOS data set (right) for the limiting break positions $D=60 \mathrm{~km}$ and $D=10 \mathrm{~km}$ for $I_{\mathrm{ex}}=0,10$, and 100 . For a given excess intensity, we can constrain the location of the break: in the absence of the excess break diameters smaller than $D_{\mathrm{b}}=16.5 \mathrm{~km}$ are ruled out; break diameters $D_{\mathrm{b}}<32.6 \mathrm{~km}$ are ruled out for $I_{\mathrm{ex}}=10$ and $D_{\mathrm{b}}<75.3 \mathrm{~km}$ are ruled out for an $I_{\mathrm{ex}}=100$ excess. When moving the excess toward smaller sizes $\left(\mu_{\mathrm{ex}}=1.6 \mathrm{~km}\right)$, break locations $D_{\mathrm{b}}<28.3 \mathrm{~km}$ are ruled out for $I_{\mathrm{ex}}=10$, and $D_{\mathrm{b}}<63.3 \mathrm{~km}$ for $I_{\mathrm{ex}}=100$.

Since the KB04 simulations show that models with weaker KBOs and Neptune stirring produce a location of the break at smaller sizes, our result strongly favors models that incorporate the effects of Neptune stirring and weaker KBOs, where the bulk strength $Q_{b} \lesssim 10^{3} \mathrm{erg} \mathrm{g}^{-1}$.

\subsection{Benavidez \& Campo Bagatin (2009)}

Recent simulations by Benavidez \& Campo Bagatin (2009, hereinafter BCB09) divide the Kuiper Belt into three dynamical families-the classical belt (CB), the Plutinos (resonant population), and the scattered disk (SD) - and follow the collisional evolution of each, while taking into account the physics of the fragmentation of icy and rocky bodies at the typical relative velocities of KBOs. This suite of models ignores the effects of Neptune stirring. The models incorporate four scaling
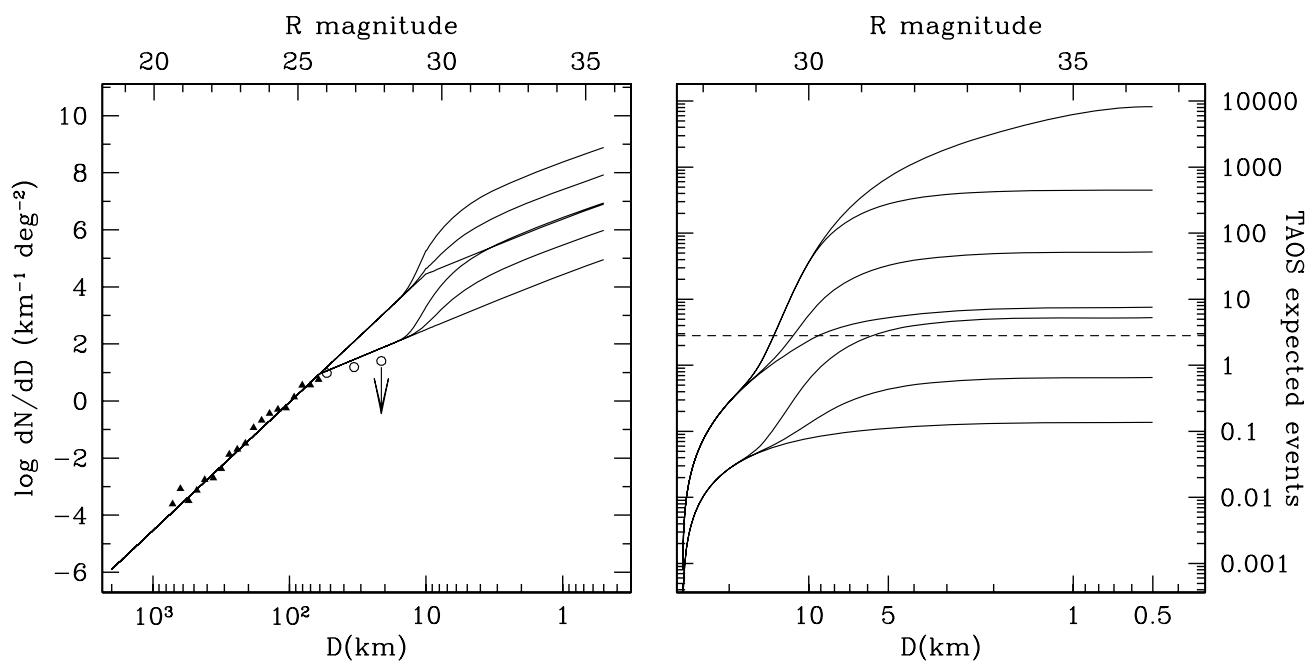

Figure 13. KB04 models. The left panel shows the differential size distribution, parameterized as two slopes $\left(q_{\mathrm{L}}=4.5\right.$ and $\left.q_{\mathrm{I}}=2\right)$ and on the right side are the corresponding event rates for the TAOS survey. Symbols are the same as in Figure 11. For limiting break positions of $D_{\mathrm{b}}=60$ and $10 \mathrm{~km}$ the distribution is plotted for a model with no Gaussian excess, and models with Gaussian excess of intensity $I_{\mathrm{ex}}=10$ and $I_{\mathrm{exp}}=100$, centered at $\mu_{\mathrm{ex}}=5.5 \mathrm{~km}$. 

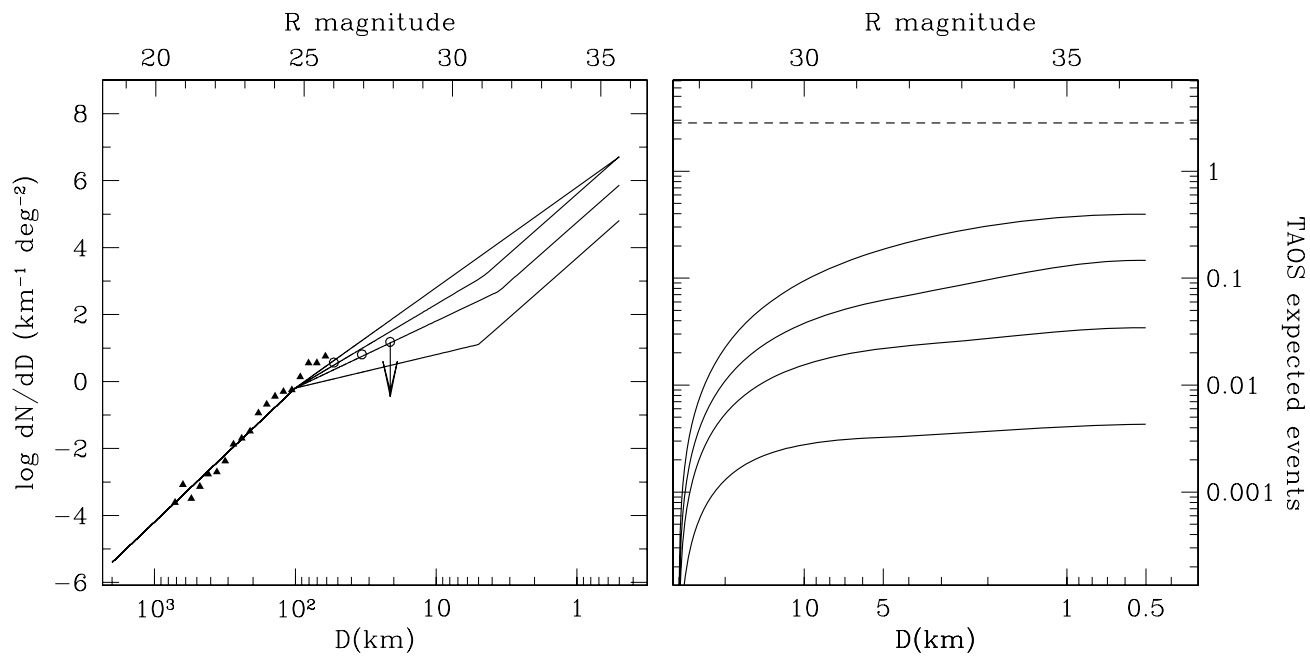

Figure 14. Our rendering of BCB09 models: the models differ in the prescription for fragmentation and all models are parameterized as a series of three slopes. Symbols are the same as in Figure 11. The first break point is fixed at $100 \mathrm{~km}$. The slope for the smallest size objects is set to 3.7. The locations of the second break and intermediate slope are $5 \mathrm{~km}$ and 1.0,3.6 km and 2.0, 4.6 km and 2.5, and $0.36 \mathrm{~km}$ and 3.0. Right: corresponding number of events in our TAOS survey.

laws for fragmentation: a simple scaling driven by gravitational self-compression, two scaling laws which include both self-compression and the effects of strain rate, as described by Farinella et al. (1982), with different diameter dependences $\left(D^{-0.25}\right.$ and $\left.D^{-0.5}\right)$ and one that follows the modeling of Benz \& Asphaug (1999) for icy bodies. They then vary the material strength of the objects between $10^{5}$ and $10^{7} \mathrm{erg} \mathrm{cm}^{-3}$.

In BCB09, the size distribution of large objects is set to a power law with slope $q_{\mathrm{L}} \approx 4.0$. This is set as an initial condition to their simulations, and since the large objects are not undergoing many collisions, this shape is preserved. A first break is seen around $100 \mathrm{~km}$. The size distribution then departs from power-law behavior for all parameter choices, with 2-4 orders of magnitudes fewer KBOs in the $\sim 1 \mathrm{~km}$ region than the nominal power law would predict. The size distribution then follows again a power law, with slope $q_{\mathrm{S}}=3.5$, in the strength-dominated regime. The BCB09 models are shown in Figure 14, left. We simplified the size distribution behavior in the intermediate region with a single power law, though typically the behavior is oscillatory. The oscillations are negligible for our predictions for all but the model which includes self-compression and the effects of strain rate with diameter dependence $D^{-0.5}$, where the inclusion of the oscillations may change the event rate by a factor of a few. This model however predicts the smallest event rate, well below the sensitivity of the TAOS data. Our conclusions are thus unaffected by this simplification. Our scaling leads to $N(D \geqslant 100 \mathrm{~km}) \sim$ $21 \mathrm{deg}^{-2}$ for the BCB09 models.

These models are all allowed by the TAOS data, partly because of the location of the initial break at $D_{\mathrm{b}}=100$ and the slope of the large end size distribution. The break location is at the large end of what is allowed by direct observations (indeed outside of the Fraser \& Kavelaars 2008 allowed range of $D_{\mathrm{b}} \in[50,95 \mathrm{~km}]$ with assumption of a $6 \%$ albedo). The location of this first break does not evolve in the BCB09 simulations from what is set as an initial condition. Similarly, the choice of a slope at the large end of the size distribution of $q_{\mathrm{L}}=4.0$ is slightly shallower than the current best-fit value from Fraser \& Kavelaars (2009) and Fuentes \& Holman (2008). As the parameters relative to the large size end of the distribution are more firmly pinned down by direct observations, these models and future occultation data may place stronger constraints on the details of the shape of the size distribution below the first break and thus the details of the fragmentation mechanisms, providing information on the internal structure of the KBOs.

\subsection{Fraser (2009)}

Fraser (2009, hereinafter F09) considers the collisional evolution of the Kuiper Belt size distribution after the epoch of accretion. Starting with initial conditions that reproduce the observed large end size distribution $\left(q_{\mathrm{L}}=4.8\right)$, which will not further evolve in the collisional simulations, and $D_{\mathrm{b} 1}=2 \mathrm{~km}$, the population is collisionally evolved over the age of the solar system. A depletion, or divot, forms at $D \sim 10 \times D_{\mathrm{b} 1}$, or $D \sim 20 \mathrm{~km}$. The size of this depletion changes when changing parameters relative to the internal strength of the KBOs or the impact velocity, as well as choices for the initial intermediate slope. An excess is also evident at smaller sizes: $D \sim 4 \mathrm{~km}$. We model the F09 size distributions as they appear in Figure 2 of F09. Our size distributions are created as a sequence of three slopes: $q_{\mathrm{L}}=4.8,1 \leqslant q_{\mathrm{I}} \leqslant 3$, and $2.5 \leqslant q_{\mathrm{S}} \leqslant 4.5$, and we model the divots and excesses with Gaussians (Figure 15, left). Here we scale the differential size distribution so that $d N / d D(D=200 \mathrm{~km}) \sim 0.047 \mathrm{deg}^{-1} \mathrm{~km}^{-1}$, to reproduce the number of objects at $D=50 \mathrm{~km} .{ }^{26}$ Our survey cannot constrain these models: the pronounced divot at $D \sim 20 \mathrm{~km}$ causes a very low event rate in our survey (Figure 15, right).

\subsection{Generic Three-regime Model: Constraints on the Intermediate Region of the Size Spectrum}

A simple, generic three-regime model allows us to describe separately the primordial region, the intermediate region and the fully collisional, strength-dominated regime. Knowing that both the large (gravitationally dominated) and the small (strengthdominated) regions of the size spectrum are relatively insensitive to the details of the internal structure and evolution of the Kuiper Belt, it is the intermediate region that contains the most information about the physical details of the KBOs. We grossly simplify the expected structure in the intermediate regime and describe it with a power law. The parameters of one such

\footnotetext{
${ }^{26} N(D=50 \mathrm{~km}) \sim 15$ (W. C. Fraser 2009, private communication).
} 

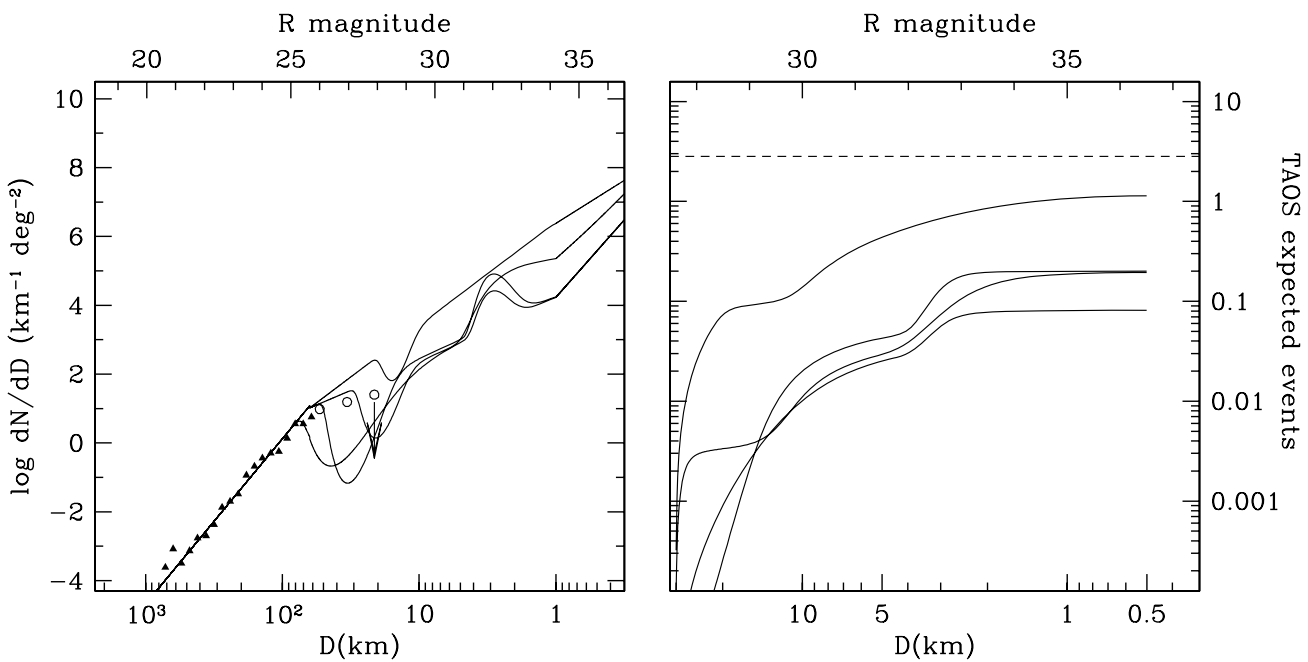

Figure 15. Left: our modeling of the F09 models. The models are reproduced on the basis of Figure 2 in F09, and divots and excesses are generated with Gaussians. Symbols are the same as in Figure 11. The slope on the large end side $(D \gtrsim 65 \mathrm{~km})$ is $q_{\mathrm{L}}=4.8$. Right: corresponding event rates for our survey.

\section{$\mathrm{R}$ magnitude}
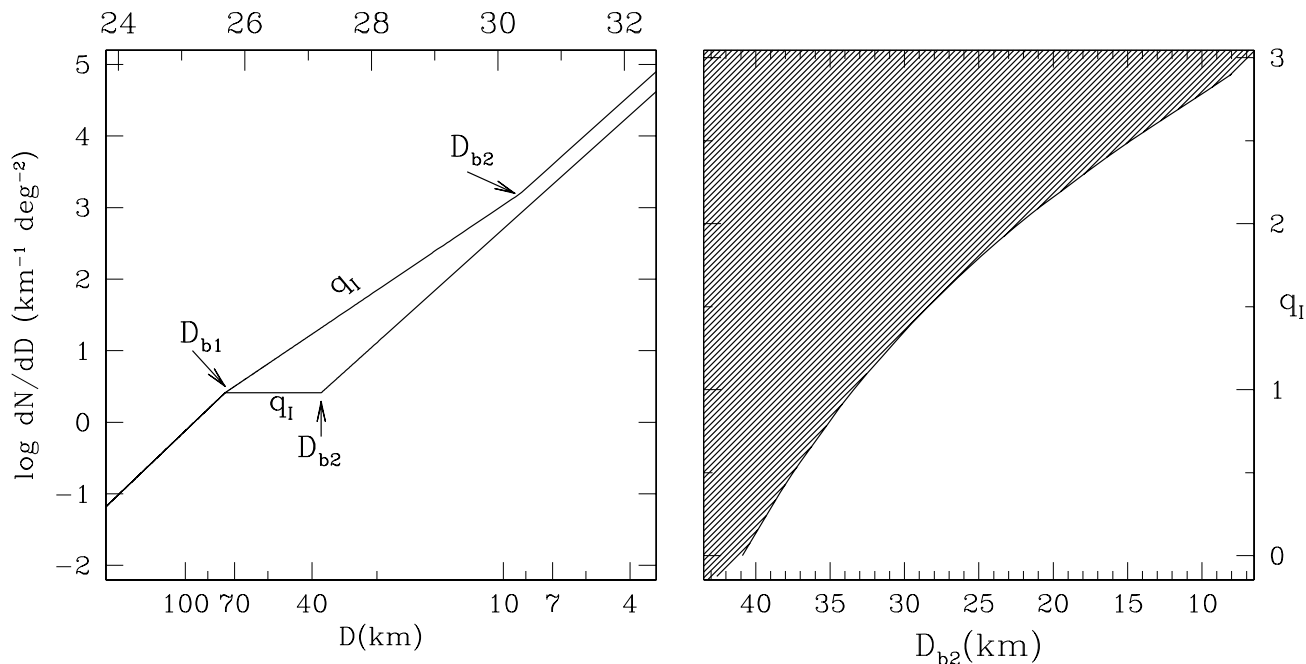

Figure 16. Three-slope model described in Section 5.5 (left) for the minimum second break location $D_{\mathrm{b} 2}$ allowed by slopes $q_{I}=0$ and 3 . Slope-break location phase space for the three-slopes model (right). The shaded region is excluded by our data.

models would then be three slopes $q_{\mathrm{L}}, q_{\mathrm{I}}$, and $q_{\mathrm{S}}$, and two break locations $D_{\mathrm{b} 1}$ and $D_{\mathrm{b} 2}$.

We set the slope of the large end of the size spectrum and the location of the first break to the best fit to the data from direct observations: $q_{\mathrm{L}}=4.8$ and $D_{\mathrm{b} 1}=75 \mathrm{~km}$. We model the intermediate region as a plateau, or with a shallow slope: $q_{\mathrm{I}} \in[0,3]$, and the small size end of the spectrum as a power law with slope 3.5 for the strength-dominated, collisionally evolved population. Figure 16 shows our three-regime model (left) and the limit we can set to the intermediate slope, $q_{\mathrm{I}}$, and second break location, $D_{\mathrm{b} 2}$, phase space (right). Any pair of values $q_{\mathrm{I}}$ and $D_{\mathrm{b} 2}$ that fall above the solid line is excluded.

\section{THE JUPITER FAMILY COMETS PROGENITOR POPULATION}

The JFCs are believed to originate in the outer solar system. In this scenario, the giant planets generate gravitational perturbations that affect the orbits of the outer solar system bodies, injecting them into the planetary region, where they are captured by Jupiter. The orbital inclination of the JFCs suggests that their precursor population has a disk-like distribution, favoring thus the Kuiper Belt over the Oort Cloud as a reservoir (Volk \& Malhotra 2008, and references therein). The CB, the Plutinos, and the SD have been considered as precursors in various studies. The dynamical characteristics of each population determines the efficiency of the injection process and the number of objects in each progenitor family can thus be derived on the basis of the density of JFCs, which is observationally constrained (see Tancredi et al. 2006, and references therein). Furthermore the size distribution of JFCs should reflect the size distribution of the progenitor population. Bernstein et al. (2004) and Bianco et al. (2009) have pointed out that a better determination of the size distribution of the Kuiper Belt would help understand the origin of the JFCs.

We assume the JFC precursors are in the size range 1-10 km as this is observed to be the typical size of JFCs (Lowry et al. 2008). We considered the estimates on the KBO populations (CB and Plutinos) and SD derived from dynamical simulations under the assumption that each population is the unique progenitor of 
the JFCs, and we compared them to the upper limits derived from our survey (Figure 9). We use the estimate of Levison \& Duncan (1997) for a population of cometary precursors entirely in the CB, and that of Morbidelli (1997) for Plutino progenitors. These are converted into a surface density by assuming for each population a projected sky area of $10^{4} \mathrm{deg}^{2}$, as was done by Bernstein et al. (2004). We consider the results of Volk \& Malhotra (2008) for a progenitor population in the SD. We calculate the minimum surface density of SD objects expected in the region of sky typically observed by the TAOS survey. For this we use information on the fraction of time the objects spend between 30 and $50 \mathrm{AU}$ and within $3^{\circ}$ of the ecliptic plane as provided by Volk \& Malhotra (2008). These estimates on the number of objects are shown in Figure 9 as horizontal lines.

Our results rule out a precursor family composed uniquely of CB objects with $D \gtrsim 3 \mathrm{~km}$. Occultation surveys are the only surveys that at present can probe this region of the size spectrum, and our preliminary result shows that future occultation surveys will be able to derive useful constraints on the origin of the JFCs.

\section{CONCLUSIONS}

We presented an analysis of 3.75 years of TAOS data, comprising $5 \times 10^{5}$ star hours of light curves sampled at 4 or $5 \mathrm{~Hz}$ observed with three telescopes simultaneously. We searched for occultations of our target stars by KBOs in order to constrain the size distribution of KBOs, particularly in the $500 \mathrm{~m}-10 \mathrm{~km}$ region, which is currently out of reach of direct observation surveys. More than $90 \%$ of the TAOS data are collected within $5^{\circ}$ of ecliptic latitude in order to maximize the occultation rate by objects in the Kuiper Belt. Occultations near opposition lead to a higher event rate for TAOS, even after taking into account the increased recovery efficiency for small objects where the angle from opposition is larger and the relative velocity of the KBOs is lower.

We found no occultation events in our data. This allowed us to set upper limits to the number density of KBOs that are stringent enough to be compared usefully with models for the formation and evolution of the Kuiper Belt. We considered four theoretical models, PS05, KB04, BCB09, and F09, all of which describe the present size distribution of the Kuiper Belt, and we set constraints on these models. This is the first detailed comparison of occultation data with specific model results.

Our result, particularly when compared with PS05 and KB04, suggests that the Kuiper Belt is populated by fragile bodies, and that the effect of the migration of Neptune played an important role in its formation. None of the BCB09 or F09 models can be ruled out.

Using a generic model, where the size distribution is described by three consecutive power laws, and fixing the slope on the large end size and the location of the first break to the best fit from direct observations $\left(q_{\mathrm{L}}=4.8 \mathrm{~km}, D_{\mathrm{b} 1}=75 \mathrm{~km}\right)$, and the slope of the small side to $q_{\mathrm{s}}=4.0$ we can constrain the intermediate slope $q_{\mathrm{I}}$ and the location of the second break $D_{\mathrm{b} 2}$, as shown in Figure 16. As direct surveys are currently not sensitive to KBOs smaller than $D \sim 28 \mathrm{~km}$ occultation surveys provide the only probe of this region of the size spectrum, and the large TAOS data set allowed us to set the first constraints to the location of the second break.

We also considered the JFCs. Assuming the JFCs are injected into their present orbit from one of the Kuiper Belt populations, $\mathrm{CB}$, Plutinos, or from the $\mathrm{SD}$, we compared the upper limit derived from our survey to the estimates of the number of objects derived using the number of JFCs by Levison \& Duncan (1997) for a population of cometary precursors entirely in the CB, that of Morbidelli (1997) for Plutinos and of Volk \& Malhotra (2008) for a progenitor population in the SD. We can rule out the unique precursor family composed of CB objects $D \gtrsim 3 \mathrm{~km}$. This preliminary result confirms that occultation surveys can help understanding the origin of JFCs.

A recent analysis of the HST guiding data (S09) reports the detection of an occultation by a $D \sim 1 \mathrm{~km} \mathrm{KBO}$. This object is detected at high ecliptic latitude $\left(b \sim 14^{\circ}\right)$. The surface density derived in S09, assuming a straight power-law size distribution for small KBOs, is within the upper limit set by TAOS, although a $1 \sigma$ increment over the best-fit surface density is ruled out to better than $95 \%$ c.l.

TAOS has operated for over four years observing continuously with three, and now four, $50 \mathrm{~cm}$ aperture telescopes. TAOS is only marginally sensitive to subkilometer KBO occultations, but we were able to prove that the low sensitivity at subkilometer sizes is more than compensated for by the vast exposure of which TAOS is capable. Improvements in our selection criteria and the analysis of four telescope data should increase our sensitivity to smaller objects.

The authors thank Scott Kenyon, for insightful conversations. Work at the CfA was supported in part by the NSF under grant AST-0501681 and by NASA under grant NNG04G113G. Work at NCU was supported by the grant NSC 96-2112-M008-024-MY3. Work at ASIAA was supported in part by the thematic research program AS-88-TP-A02. Work at Yonsei was supported by National Research Foundation of Korea through grant 2009-0075376 (Space Science Institute). The work of N. Coehlo was supported in part by NSF grant DMS-0636667. Work at LLNL was performed in part under USDOE Contract W-7405-Eng-48 and Contract DE-AC52-07NA27344. Work at SLAC was performed under USDOE contract DE-AC0276SF00515. Work at NASA Ames was supported by NASA's Planetary Geology \& Geophysics Program.

\section{REFERENCES}

Benavidez, P. G., \& Campo Bagatin, A. 2009, Planet. Space Sci., 57, 201

Benz, W., \& Asphaug, E. 1999, Icarus, 142, 5

Bernstein, G. M., et al. 2004, AJ, 128, 1364

Bianco, F. B., et al. 2009, AJ, 138, 568

Bickerton, S. J., Kavelaars, J. J., \& Welch, D. L. 2008, AJ, 135, 1039

Bickerton, S. J., Welch, D. L., \& Kavelaars, J. J. 2009, AJ, 137, 4270

Born, M., \& Wolf, E. 1980, Principles of Optics. Electromagnetic Theory of Propagation, Interference and Diffraction of Light (6th corrected Oxford: Pergamon)

Chang, H.-K., et al. 2007, MNRAS, 378, 1287

Charnoz, S., \& Morbidelli, A. 2007, Icarus, 188, 468

Cutri, R. M., et al. 2003, 2MASS All Sky Catalog of Point Sources (Pasadena, CA: IPAC)

Davis, D. R., \& Farinella, P. 1997, Icarus, 125, 50

Dohnanyi, J. W. 1969, J. Geophys. Res., 74, 2531

Farinella, P., Paolicchi, P., \& Zappala, V. 1982, Icarus, 52, 409

Fraser, W. C. 2009, ApJ, 706, 119

Fraser, W. C., \& Kavelaars, J. J. 2008, Icarus, 198, 452

Fraser, W. C., \& Kavelaars, J. J. 2009, AJ, 137, 72

Fuentes, C. I., George, M. R., \& Holman, M. J. 2009, ApJ, 696, 91

Fuentes, C. I., \& Holman, M. J. 2008, AJ, 136, 83

Kenyon, S. J., \& Bromley, B. C. 2001, AJ, 121, 538

Kenyon, S. J., \& Bromley, B. C. 2004, AJ, 128, 1916

Kenyon, S. J., \& Luu, J. X. 1999a, AJ, 118, 1101

Kenyon, S. J., \& Luu, J. X. 1999b, ApJ, 526, 465 
Kenyon, S. J., \& Windhorst, R. A. 2001, ApJ, 547, L69

Kenyon, S. J., et al. 2008, in The Solar System Beyond Neptune, Formation and Collisional Evolution of Kuiper Belt Objects, ed. M. A. Barucci, H. Boehnhardt, D. P. Cruikshank, \& A. Morbidelli (Tucson, AZ: Univ. Arizona Press), 293

Lehner, M. J., et al. 2009, PASP, 121, 138

Lehner, M. J., et al. 2010, PASP, submitted (arXiv:1002.3626v1)

Leinhardt, Z. M. 2008, in The Solar System Beyond Neptune, Physical Effects of Collisions in the Kuiper Belt, ed. M. A. Barucci, H. Boehnhardt, D. P. Cruikshank, \& A. Morbidelli (Tucson, AZ: Univ. Arizona Press), 195

Levison, H. F., \& Duncan, M. J. 1997, Icarus, 127, 13

Liang, C. L., et al. 2004, Stat. Sci., 19, 265

Liu, C.-Y., et al. 2008, MNRAS, 388, L44

Lowry, S., et al. 2008, in The Solar System Beyond Neptune, Kuiper Belt Objects in the Planetary Region: The Jupiter-Family Comets, ed. M. A. Barucci, H. Boehnhardt, D. P. Cruikshank, \& A. Morbidelli (Tucson, AZ: Univ. Arizona Press), 397

Monet, D. G., et al. 2003, AJ, 125, 984

Morbidelli, A. 1997, Icarus, 127, 1

Morbidelli, A., Levison, H. F., \& Gomes, R. 2008, in The Solar System Beyond Neptune, The Dynamical Structure of the Kuiper Belt and Its Primordial
Origin, ed. M. A. Barucci, H. Boehnhardt, D. P. Cruikshank, \& A. Morbidelli (Tucson, AZ: Univ. Arizona Press), 275

Moro-Martin, A., et al. 2008, in The Solar System Beyond Neptune, Extra Solar Kuiper Belt Dust Disks, ed. M. A. Barucci, H. Boehnhardt, D. P. Cruikshank, $\&$ A. Morbidelli (Tucson, AZ: Univ. Arizona Press), 465

Nihei, T. C., et al. 2007, AJ, 134, 1596

Nordgren, T. E., et al. 2002, AJ, 123, 3380

Pan, M., \& Sari, R. 2005, Icarus, 173, 342

Roques, F., \& Moncuquet, M. 2000, Icarus, 147, 530

Roques, F., Moncuquet, M., \& Sicardy, B. 1987, AJ, 93, 1549

Roques, F., et al. 2006, AJ, 132, 819

Schenk, P. M., \& Zahnle, K. 2007, Icarus, 192, 135

Schlichting, H. E., et al. 2009, Nature, 462, 895

Stern, S. A. 1996, AJ, 112, 1203

Tancredi, G., et al. 2006, Icarus, 182, 527

Tsiganis, K., et al. 2005, Nature, 435, 459

van Belle, G. T. 1999, PASP, 111, 1515

Volk, K., \& Malhotra, R. 2008, ApJ, 687, 714

Wang, J.-H., et al. 2009, AJ, submitted (arXiv:0910.5598)

Zhang, Z.-W., et al. 2008, ApJ, 685, L157

Zhang, Z. W., et al. 2009, PASP, 121, 1429 